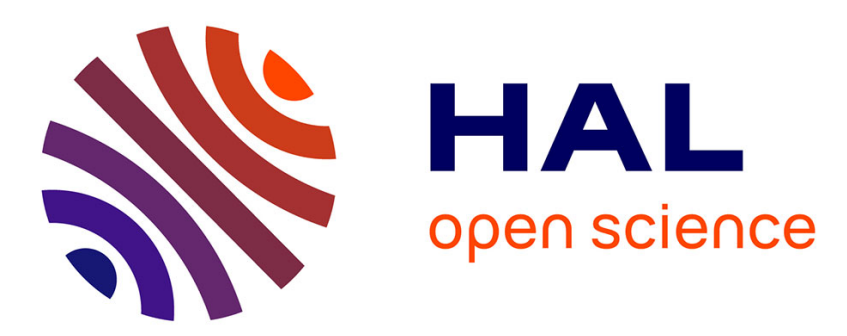

\title{
System-level analysis of receiver diversity in SWIPT-enabled cellular networks
}

Lam Thanh Tu, Marco Di Renzo, Justin P. Coon

\section{To cite this version:}

Lam Thanh Tu, Marco Di Renzo, Justin P. Coon. System-level analysis of receiver diversity in SWIPT-enabled cellular networks. Journal of Communications and Networks, 2016, 18 (6), pp.926 937. 10.1109/JCN.2016.000127 . hal-01880124

\section{HAL Id: hal-01880124 https://hal.science/hal-01880124}

Submitted on 7 Jul 2020

HAL is a multi-disciplinary open access archive for the deposit and dissemination of scientific research documents, whether they are published or not. The documents may come from teaching and research institutions in France or abroad, or from public or private research centers.
L'archive ouverte pluridisciplinaire HAL, est destinée au dépôt et à la diffusion de documents scientifiques de niveau recherche, publiés ou non, émanant des établissements d'enseignement et de recherche français ou étrangers, des laboratoires publics ou privés. 


\title{
System-Level Analysis of Receiver Diversity in
}

\section{SWIPT-Enabled Cellular Networks}

\author{
Thanh Tu Lam, Student Member, IEEE, Marco Di Renzo, Senior Member, IEEE, \\ and Justin P. Coon, Senior Member, IEEE
}

\begin{abstract}
In this paper, we study the feasibility of receiver diversity for application to downlink cellular networks, where low-energy devices are equipped with information decoding and energy harvesting receivers for simultaneous wireless information and power transfer. We compare several options that are based on selection combining and maximum ratio combining, which provide different implementation complexities. By capitalizing on the Frechet inequality, we shed light on the advantages and limitations of each scheme as a function of the transmission rate and harvested power that need to be fulfilled at the low-energy devices. Our analysis shows that no scheme outperforms the others for every system setup. It suggests, on the other hand, that the low-energy devices need to operate in an adaptive fashion, by choosing the receiver diversity scheme as a function of the imposed requirements. With the aid of stochastic geometry, we introduce mathematical frameworks for system-level analysis. We show that they constitute an important tool for system-level optimization and, in particular, for identifying the diversity scheme that optimizes wireless information and power transmission as a function of a sensible set of parameters. Monte Carlo simulations are used to validate our findings and to illustrate the trade-off that emerge in cellular networks with simultaneous wireless information and power transfer.
\end{abstract}

\section{Index Terms}

Cellular Networks, Simultaneous Wireless Information and Power Transfer, Stochastic Geometry.

Manuscript received March 24, 2016; revised June 26, 2016.

T. Tu Lam and M. Di Renzo are with the Laboratoire des Signaux et Systèmes, CNRS, CentraleSupélec, Univ Paris Sud, Université Paris-Saclay, 3 rue Joliot Curie, Plateau du Moulon, 91192, Gif-sur-Yvette, France. (e-mail: lamthanh.tu@12s.centralesupelec.fr, marco.direnzo@12s.centralesupelec.fr).

J. P. Coon is with the Department of Engineering Science, Oxford University, Parks Road, Oxford, OX1 3PJ, United Kingdom. (e-mail: justin.coon@eng.ox.ac.uk). 


\section{INTRODUCTION}

The Internet of Things (IoT) is expected to connect billions of Low-Energy Devices (LEDs) by 2020 [1]. One of the main challenges of the IoT is how to provide enough energy for the electronics of the LEDs, in order to have them operational over a reasonable amount of time and without making either their battery too large or the device itself too bulky. For several applications, it may not be even possible to (re-)charge some kinds of LEDs.

In this context, the emerging concept of Simultaneous Wireless Information and Power Transfer (SWIPT) constitutes a suitable solution for prolonging the battery life of the LEDs and, in a foreseeable future, for making them energy-neutral, i.e., operational in a complete self-powered fashion. SWIPT, more specifically, is a technology where the same radio frequency signal is used for information transmission and for replenishing the battery of the LEDs [2]. SWIPT may find application in the emerging market of cellular-enabled IoT, where the LEDs, e.g., smart watches [3], receive notifications from their cellular connection [4] and, simultaneously, re-charge their battery. The recent decision to standardize NarrowBand IoT (NB-IoT), a new narrow-band radio technology that addresses the requirements of the IoT, confirms the wish of capitalizing on the ubiquitous coverage offered by the cellular network infrastructure for IoT applications [5].

The design of SWIPT-enabled cellular networks introduces, however, new research challenges and never observed trade-offs. Conventional cellular networks are designed based on the assumption that the interference has a negative impact on Information Decoding (ID), since it reduces the coverage and rate [6]. The same interference, on the other hand, is a natural source of power for Energy Harvesting (EH) [7]. As a result, the development of interference management techniques that exploit interference for $\mathrm{EH}$ and counteract it for ID plays a fundamental role. In this context, receiver diversity is considered to be a promising solution for enhancing the reliability of data transmission and for increasing the amount of harvested power [8]. The size of the LEDs, in fact, is expected to be larger than that of sensor nodes, and, as a consequence, multiple radiating (antenna) elements may be available. On the other hand, their size, cost and power consumption requirements may still limit the number of radio frequency front-ends that may be used [9]. Receiver diversity constitutes a practical solution for taking advantage of the available antenna elements by using fewer radio frequency front-ends. It provides, in fact, the 
possibility of optimizing performance, cost and power consumption for a given size of the LEDs.

The potential of receiver diversity for application to SWIPT-enabled systems has recently been analyzed in [10] and [11]. In these papers, in particular, it has been shown that receiver diversity based on selection combining and antenna switching constitutes a promising alternative to typical approaches based on power splitting and time switching [12]. As elaborated in [11], in fact, power splitting and time switching need dedicated hardware components (power splitters and time switches), which may increase the complexity and cost of the LEDs and may be subject to efficiency losses. Time switching, in addition, necessitates dedicated time slots and synchronization circuits for $\mathrm{EH}$, which results in the discontinuous transmission of information data. Receiver diversity, on the other hand, is a mature technology that may overcome these limitations. It requires, however, the availability of multiple antenna elements at the LEDs. This leads to new performance versus implementation complexity trade-offs (further details are available in Section III-A) that, to the best of the authors' knowledge, are not totally understood.

Motivated by these considerations, we study the potential of receiver diversity for application to SWIPT-enabled cellular networks. In particular, we focus our attention on practical implementations where one or two receive antennas are available at the LEDs. This implies that only one or two radio frequency front-ends are needed. This case study may find concrete application to LEDs such as smart watches, since the typical circumference of a human wrist is $14-20 \mathrm{~cm}$, and, thus, two compact integrated antennas and radio frequency front-ends may be accommodated at typical transmission frequencies. More antenna elements may be used, by still employing one or two radio frequency chains, for LEDs with larger size, e.g., for relay nodes [8]. We study various options based on selection combining and maximum ratio combining schemes, and discuss their achievable performance versus implementation complexity trade-off. Our analysis, in particular, shows that no scheme outperforms the others for every system setup. It suggests, on the other hand, that the LEDs need to operate in an adaptive fashion, by choosing the receiver diversity scheme to be used as a function of the performance requirements that need to be fulfilled.

Against the state-of-the-art of research on performance evaluation of wireless networks with SWIPT, our contribution is twofold. Compared to [10] and [11], we focus our attention on systemlevel analysis and optimization rather than on link-level optimization. More specifically, we take 
into account the impact of large-scale network deployments and introduce new mathematical frameworks for the adaptive optimization of SWIPT-enabled cellular networks. This is performed by exploiting the mathematical tool of stochastic geometry and by modeling the locations of cellular Base Stations (BSs) as points of a Poisson Point Process (PPP). Compared to recent papers that have exploited similar mathematical tools for large-scale analysis of wireless networks with SWIPT, e.g., [13]- [18], our paper is the first that investigates the potential of receiver diversity for application to cellular networks. In [13]- [17], on the other hand, decentralized (ad hoc) networks without receiver diversity are studied. In [18], cellular networks are analyzed but single-antenna LEDs are considered. The latter paper constitutes, however, the benchmark against which the potential benefits of receiver diversity studied in this paper are quantified.

The remainder of this paper is organized as follows. In Section II, the system model is introduced. In Section III, the problem at hand is first motivated and then formulated in terms of the Joint Complementary Cumulative Distribution Function (J-CCDF) of information rate and harvested power. In Section IV, several SWIPT schemes are compared against each other with the aid of the Frechet inequality. In Section V, mathematical frameworks for the system-level performance evaluation and for the adaptive optimization of SWIPT-enabled cellular networks are introduced. In Section VI, analysis and findings are validated with the aid of numerical simulations. Finally, Section VII concludes this paper.

Notation: Main notation and most recurrent mathematical symbols are reported in Table I.

\section{SYSTEM MODEL}

\section{A. Cellular Networks Modeling}

A downlink cellular network is considered. The BSs are modeled as points of a homogeneous PPP, denoted by $\Psi$, of density $\lambda$. The transmit power of the BSs is assumed to be fixed and is denoted by $P$. Without loss of generality, the mathematical analysis is performed for the typical LED located at the origin [6]. 
TABLE I: Main notation and mathematical symbols.

\begin{tabular}{|c|c|}
\hline Symbol & Definition \\
\hline$(\cdot)^{*}$ & Conjugate operator \\
\hline$j=\sqrt{-1}$ & Imaginary unit \\
\hline $\mathbb{E}\{\cdot\}$ & Expectation operator \\
\hline $\operatorname{Pr}\{\cdot\}$ & Probability measure \\
\hline$\cup$ & Union of sets \\
\hline $\operatorname{Im}\{\cdot\}$ & Imaginary part operator \\
\hline $\min \{\cdot, \cdot\}, \max \{\cdot, \cdot\}$ & Minimum and maximum operators \\
\hline $\mathbf{1}(\cdot)$ & Indicator function \\
\hline$\delta(\cdot)$ & Dirac delta function \\
\hline $\mathcal{H}(\cdot)$ & Heaviside function \\
\hline$\overline{\mathcal{H}}(x)=1-\mathcal{H}(x)$ & Complementary Heaviside function \\
\hline${ }_{p} F_{q}\left(a_{1}, \ldots, a_{p} ; b_{1}, \ldots, b_{q} ; \cdot\right)$ & Generalized hypergeometric function \\
\hline$\Gamma(\cdot, \cdot)$ & Upper-incomplete gamma function \\
\hline$f_{X}(\cdot)$ & Probability Density Function (PDF) of Random Variable (RV) $X$ \\
\hline$F_{X}(\cdot)$ & Cumulative Distribution Function (CDF) of RV $X$ \\
\hline$\Phi_{X}(\cdot)$ & Characteristic Function (CF) of RV $X$ \\
\hline $\mathcal{M}_{X}(\cdot)$ & Moment Generating Function (MGF) of RV $X$ \\
\hline$X \sim \mathcal{E}(\mu)$ & $X$ is a RV whose PDF is $f_{X}(\xi)=\mu \exp (-\mu \xi)$ \\
\hline$X \sim \mathcal{E}_{\max }(1)$ & $X$ is a RV whose PDF is $f_{X}(\xi)=2 \exp (-\xi)-2 \exp (-2 \xi)$ \\
\hline$X \sim \mathcal{G}(2,1)$ & $X$ is a RV whose PDF is $f_{X}(\xi)=\xi \exp (-\xi)$ \\
\hline$X \stackrel{d}{=} Y$ & The RVs $X, Y$ are equivalent in distribution, i.e., their CFs and MGFs are the same \\
\hline $\mathcal{R}, \mathcal{Q}$ & Information rate, harvested power \\
\hline $\mathcal{R}_{*}, \mathcal{Q}_{*}$ & Information rate, energy harvesting requirements \\
\hline $\bar{F}(\cdot, \cdot)$ & Joint Complementary Cumulative Distribution Function (J-CCDF) of $\mathcal{R}$ and $\mathcal{Q}$ \\
\hline$q_{s}^{[a, b]}$ & Probability that a link of length $r \in[a, b)$ is in state $s$ \\
\hline$D$ & Line-of-Sight (LOS) / Non-LOS (NLOS) ball \\
\hline$L^{(0)}$ & Smallest path-loss \\
\hline$\Psi, \Psi_{s}$ & Poisson Point Process (PPP) of Base Stations (BSs), PPP of BSs in state $s$ \\
\hline$\lambda, \lambda_{s}$ & Density of BSs, density of BSs in state $s$ \\
\hline$P$ & Transmit power of BSs \\
\hline$\beta_{s}, \kappa_{0}$ & Path-loss exponent of links in state $s$, free-space path-loss constant \\
\hline$G_{\mathrm{M}}, G_{\mathrm{S}}$ & Beamforming gains of main $(\mathrm{M})$ and side $(\mathrm{S})$ lobes \\
\hline$\theta_{\mathrm{M}}$ & Beamwidth of main lobe \\
\hline $0<\rho<1$ & Power splitting ratio \\
\hline$B_{w}$ & Transmission bandwidth \\
\hline$\sigma_{\mathrm{N}}^{2}$ & Thermal noise power \\
\hline$\sigma_{\mathrm{ID}}^{2}$ & Noise power due to the signal conversion from radio frequency to baseband \\
\hline $0 \leq \zeta \leq 1$ & Efficiency of energy harvesting conversion \\
\hline$r_{*}=\left(2^{\mathcal{R}_{*} / B_{w}}-1\right)^{-1}, \tilde{r}_{*}=r_{*}$ & Short-hands used in Proposition 1 and Proposition 2 \\
\hline$\sigma_{*}^{2}=\sigma_{N}^{2}+\sigma_{\mathrm{ID}}^{2}(1-\rho)^{-1}, \tilde{\sigma}_{*}^{2}=\sigma_{N}^{2}+\sigma_{\mathrm{ID}}^{2} / \rho_{\mathrm{ID}}$ & Short-hands used in Proposition 1 and Proposition 2 \\
\hline$q_{*}=\mathcal{Q}_{*}(\rho \zeta)^{-1}, \tilde{q}_{*}=\mathcal{Q}_{*}\left(\rho_{\mathrm{EH}} \zeta\right)^{-1}$ & Short-hands used in Proposition 1 and Proposition 2 \\
\hline $\mathcal{T}_{*}=\left(q_{*}+\sigma_{*}^{2}\right) /\left(r_{*}+1\right), \tilde{\mathcal{T}}_{*}=\left(\tilde{q}_{*}+\tilde{\sigma}_{*}^{2}\right) /\left(\tilde{r}_{*}+1\right)$ & Short-hands used in Proposition 1 and Proposition 2 \\
\hline
\end{tabular}

\section{B. Channel Modeling}

The channel model accounts for Line-of-Sight (LOS) and Non-LOS (NLOS) links due to the presence of spatial blockages, for the path-loss, and for the fast-fading. Shadowing is implicitly taken into account via the LOS and NLOS link model [19]. 
1) LOS/NLOS Links: Let $r$ be the distance from a BS to the typical LED. The probability of LOS and NLOS as a function of $r, p_{s}(\cdot)$ for $s \in\{\mathrm{LOS}, \mathrm{NLOS}\}$, is formulated as follows:

$$
p_{s}(r)=\left\{\begin{array}{lll}
q_{s}^{[0, D]} & \text { if } & r \in[0, D) \\
q_{s}^{[D, \infty]} & \text { if } \quad r \in[D,+\infty),
\end{array}\right.
$$

where $q_{\mathrm{LOS}}^{[a, b]}+q_{\mathrm{NLOS}}^{[a, b]}=1,0 \leq q_{s}^{[a, b]} \leq 1$ is the probability that a link of length $r \in[a, b)$ is in state $s$, and $D$ takes into account that LOS and NLOS probabilities are different for short and long distances [19]. Assuming no spatial correlation among the links, $\Psi$ can be split in two independent and non-homogeneous PPPs, $\Psi_{\mathrm{LOS}}$ and $\Psi_{\mathrm{NLOS}}$, such that $\Psi=\Psi_{\mathrm{LOS}} \cup \Psi_{\mathrm{NLOS}}$. From (1) and the thinning theorem of PPPs, the density of $\Psi_{s}$ is $\lambda_{s}(r)=\lambda p_{s}(r)$ for $s \in\{$ LOS, NLOS $\}$.

2) Path-Loss: The path-loss of LOS and NLOS links is $l_{s}(r)=\kappa_{0} r^{\beta_{s}}$ for $s \in\{$ LOS, NLOS $\}$, where $\kappa_{0}=(4 \pi / \nu)^{2}, \nu$ is the transmission wavelength, and $\beta_{s}$ is the path-loss exponent.

3) Fast-Fading: The channels gains are independent and identically distributed (i.i.d.) complex Gaussian RVs with zero mean and unit variance, i.e., Rayleigh fading is considered.

\section{Cell Association}

The typical LED is served by the BS providing the smallest path-loss. The other BSs act as interferers. The smallest path-loss can be formulated as $L^{(0)}=\min \left\{L_{\mathrm{LOS}}^{(0)}, L_{\mathrm{NLOS}}^{(0)}\right\}$, where, for $s \in\{\operatorname{LOS}, \mathrm{NLOS}\}, L_{s}^{(0)}$ is the smallest path-loss of $\Psi_{s}$, which is defined as follows:

$$
L_{s}^{(0)}=\min _{n \in \Psi_{s}}\left\{l_{s}\left(r^{(n)}\right)\right\},
$$

where $r^{(n)}$ is the distance between the $n$th BS of $\Psi_{s}$ and the typical LED.

\section{Directional Beamforming at the BSs}

At the BSs, to enhance the efficiency of information transmission and energy transfer over long distances, directional beamforming is used. Compared with more sophisticated beamforming schemes [17], it has the advantage of not necessitating channel information at the BSs. Directional beamforming can be implemented by using, e.g., uniform linear arrays [18, Sec. II-C]. We consider a two-lobe model for the radiation pattern, where $\theta_{\mathrm{M}}$ is the beamwidth of the main 
lobe, and $G_{\mathrm{M}}$ and $G_{\mathrm{S}}$ are the beamforming gains of main and side lobes, respectively. The triplet $\left(\theta_{\mathrm{M}}, G_{\mathrm{M}}, G_{\mathrm{S}}\right)$ satisfies the unit power constraint, i.e., $\theta_{\mathrm{M}} G_{\mathrm{M}}+\left(2 \pi-\theta_{\mathrm{M}}\right) G_{\mathrm{S}}=2 \pi$. Due to their small form factor, the LEDs are assumed to use omnidirectional antennas with a unit gain.

The typical LED and its serving BS estimate the angles of arrival and adjust their antenna steering orientations accordingly. Thus, the antenna gain of the typical intended link is $G^{(0)}=$ $G_{\mathrm{M}}$. From the perspective of the typical LED, on the other hand, the beams of all interfering BSs are randomly oriented, i.i.d., and uniformly distributed in $[0,2 \pi)$. Thus, the PDF of the antenna gain of the $i$ th interfering link, $G^{(i)}$, can be formulated as follows:

$$
f_{G^{(i)}}(g)=\frac{\theta_{\mathrm{M}}}{2 \pi} \delta\left(g-G_{\mathrm{M}}\right)+\left(1-\frac{\theta_{\mathrm{M}}}{2 \pi}\right) \delta\left(g-G_{\mathrm{S}}\right)
$$

\section{E. SWIPT and Receiver Diversity at the LEDs}

Due to their small form factor, the LEDs cannot accommodate many receive antennas. Hence, we analyze the case studies where the number of receive antennas and radio frequency frontends, $N_{r}$, is either $N_{r}=1$ or $N_{r}=2$. These two setups find practical application to wrist-worn LEDs, e.g., smart watches, since the average circumference of a human wrist is about 14-20 $\mathrm{cm}$. The LEDs are equipped with separate units for ID and EH. To shed light on the impact of receiver diversity, five schemes for SWIPT are studied and compared.

- Power Splitting (PS). $N_{r}=1$ is assumed and the received power, $\mathrm{P}_{\mathrm{RX}}$, is split in two parts, according to a power splitting ratio $0 \leq \rho \leq 1: \mathrm{P}_{\mathrm{EH}}=\rho \mathrm{P}_{\mathrm{RX}}$ is used for $\mathrm{EH}$ and $\mathrm{P}_{\mathrm{ID}}=\mathrm{P}_{\mathrm{RX}}-\mathrm{P}_{\mathrm{EH}}=(1-\rho) \mathrm{P}_{\mathrm{RX}}$ is used for ID.

- Power Splitting with Maximum Ratio Combining (PS-MRC). $N_{r}=2$ is assumed and the signals of the two receive antennas are combined according to the MRC scheme. The power after combining, $\mathrm{P}_{\mathrm{RX}}$, is split in two parts, according to a power splitting ratio $0 \leq \rho \leq 1$ : $\mathrm{P}_{\mathrm{EH}}=\rho \mathrm{P}_{\mathrm{RX}}$ is used for $\mathrm{EH}$ and $\mathrm{P}_{\mathrm{ID}}=\mathrm{P}_{\mathrm{RX}}-\mathrm{P}_{\mathrm{EH}}=(1-\rho) \mathrm{P}_{\mathrm{RX}}$ is used for ID.

- Separate Antenna Receiver $(S A R) . N_{r}=2$ is assumed and the received power of the first and second receive antenna is sent, without loss of generality, to the input of the ID and EH unit, respectively. The two antennas can be used for ID and EH interchangeably. 
- ID-Prioritized Selection Combining (ID-SC). $N_{r}=2$ is assumed and the received power of the antenna providing the best and the worst channel power gain is sent to the input of the ID and EH unit, respectively.

- EH-Prioritized Selection Combining (EH-SC). $N_{r}=2$ is assumed and the received power of the antenna providing the best and the worst channel power gain is sent to the input of the $\mathrm{EH}$ and ID unit, respectively.

The proposed study can be generalized for application to SWIPT implementations based on the time switching scheme [18]. For brevity, this case study is not analyzed in this paper.

\section{Problem Statement}

Considered individually, the performance of ID and EH units is usually quantified in terms of information rate and harvested power, respectively. Let $\mathcal{R}$ and $\mathcal{Q}$ denote the Shannon rate (in bits/sec) of the ID unit and the harvested power (in Watts) of the EH unit, respectively. As far as the five SWIPT schemes introduced in Section II-E are concerned, $\mathcal{R}$ and $\mathcal{Q}$ can be formulated as follows:

$$
\begin{aligned}
& \mathcal{R}=B_{w} \log _{2}\left(1+\frac{P G^{(0)} \mathcal{U}_{\mathrm{ID}}^{(0)} / L^{(0)}}{P \mathcal{I}_{\mathrm{ID}}\left(L^{(0)}\right)+\sigma_{\mathrm{N}}^{2}+\sigma_{\mathrm{ID}}^{2} / \rho_{\mathrm{ID}}}\right) \\
& \mathcal{Q}=\rho_{\mathrm{EH}} \zeta\left(P G^{(0)} \mathcal{U}_{\mathrm{EH}}^{(0)} / L^{(0)}+P \mathcal{I}_{\mathrm{EH}}\left(L^{(0)}\right)\right),
\end{aligned}
$$

where the notation in Table $\mathrm{I}$ is used, and, for $z \in\{\mathrm{ID}, \mathrm{EH}\}, 0 \leq \rho_{z} \leq 1$ accounts for the amount of power at the input of ID and EH units, $\mathcal{U}_{z}^{(0)}$ is the power gain of the intended link, and $\mathcal{I}_{z}(\cdot)$ is the aggregate other-cell interference defined as follows:

$$
\mathcal{I}_{z}\left(L^{(0)}\right)=\sum_{s \in\{\mathrm{LOS}, \mathrm{NLOS}\}} \sum_{i \in \Psi_{s}} \frac{G^{(i)} \gamma_{z}^{(i)}}{l_{s}\left(r^{(i)}\right)} \mathbf{1}\left(l_{s}\left(r^{(i)}\right)>L^{(0)}\right),
$$

where $\gamma_{z}^{(i)}$ is the power gain of the $i$ th interfering BS. The definition and the distribution of the parameters $\mathcal{U}_{z}^{(0)}, \gamma_{z}^{(i)}$ and $\rho_{z}$ are summarized in Table II.

Remark 1: Based on Table II, $\mathcal{I}_{\mathrm{ID}}(\cdot)=\mathcal{I}_{\mathrm{EH}}(\cdot)$ for PS and PS-MRC schemes, but $\mathcal{I}_{\mathrm{ID}}(\cdot) \neq$ $\mathcal{I}_{\mathrm{EH}}(\cdot)$ for SAR, ID-SC and EH-SC schemes. As far as the latter three SWIPT schemes are concerned, however, $\mathcal{I}_{\mathrm{ID}}(\cdot) \stackrel{d}{=} \mathcal{I}_{\mathrm{EH}}(\cdot)$. More precisely, $\mathcal{I}_{\mathrm{ID}}(\cdot)$ and $\mathcal{I}_{\mathrm{EH}}(\cdot)$ are partially correlated 
TABLE II: Definition and distribution of $\mathcal{U}_{z}^{(0)}, \gamma_{z}^{(i)}$ and $\rho_{z}$ in (4) for the five SWIPT schemes in Section II-E. $\gamma_{r}^{(0)} \sim \mathcal{E}(1)$ and $\gamma_{r}^{(i)} \sim \mathcal{E}(1)$ are the channel power gains of intended and $i$ th interfering BSs at the $r$ th receive antenna; $\gamma_{\text {MRC }}^{(i)}, \gamma_{\max }^{(i)}$, and $\gamma_{\min }^{(i)}$ are the channel power gains of the $i$ th interfering BS after applying MRC and impinging on the best and worst (as far as the the probe link is concerned) receive antennas, respectively. Short-hands: psc $=$ power splitting circuit, $2 \mathrm{rx}=$ two receive antennas, asc $=$ antenna switching circuit, imp $=$ implementation.

\begin{tabular}{|c|c|c|c|c|c|c|c|c|c|}
\hline & \multicolumn{2}{|l|}{$\mathcal{U}_{\mathrm{ID}}^{(0)}$} & \multicolumn{2}{|l|}{$\mathcal{U}_{\mathrm{EH}}^{(0)}$} & $\gamma_{\mathrm{ID}}^{(i)}$ & $\gamma_{\mathrm{EH}}^{(i)}$ & $\rho_{\text {ID }}$ & $\rho_{\mathrm{EH}}$ & $\mathrm{imp}$ \\
\hline PS & \multicolumn{2}{|c|}{$\gamma_{1}^{(0)} \sim \mathcal{E}(1)$} & \multicolumn{2}{|c|}{$\gamma_{1}^{(0)} \sim \mathcal{E}(1)$} & $\gamma_{1}^{(i)} \sim \mathcal{E}(1)$ & $\gamma_{1}^{(i)} \sim \mathcal{E}(1)$ & $1-\rho$ & $\rho$ & psc \\
\hline PS-MRC & \multicolumn{2}{|c|}{$\gamma_{1}^{(0)}+\gamma_{2}^{(0)} \sim \mathcal{G}(2,1)$} & \multicolumn{2}{|c|}{$\gamma_{1}^{(0)}+\gamma_{2}^{(0)} \sim \mathcal{G}(2,1)$} & $\gamma_{\mathrm{MRC}}^{(i)} \sim \mathcal{E}(1)$ & $\gamma_{\mathrm{MRC}}^{(i)} \sim \mathcal{E}(1)$ & $1-\rho$ & $\rho$ & $\mathrm{psc}+2 \mathrm{rx}$ \\
\hline SAR & \multicolumn{2}{|c|}{$\gamma_{1}^{(0)} \sim \mathcal{E}(1)$} & \multicolumn{2}{|c|}{$\gamma_{2}^{(0)} \sim \mathcal{E}(1)$} & $\gamma_{1}^{(i)} \sim \mathcal{E}(1)$ & $\gamma_{2}^{(i)} \sim \mathcal{E}(1)$ & 1 & 1 & $2 \mathrm{rx}$ \\
\hline ID-SC & $\max \left\{\gamma_{1}^{(0)}, \gamma_{2}^{(0)}\right\}$ & $\sim \mathcal{E}_{\max }(1)$ & $\gamma_{1}^{(0)}, \gamma_{2}^{(0)}$ & $\sim \mathcal{E}(2)$ & $\gamma_{\max }^{(i)} \sim \mathcal{E}(1)$ & $\gamma_{\min }^{(i)} \sim \mathcal{E}(1)$ & 1 & 1 & $2 \mathrm{rx}+\mathrm{asc}$ \\
\hline EH-SC & $\gamma_{1}^{(0)}, \gamma_{2}^{(0)}$ & $\sim \mathcal{E}(2)$ & $\max \left\{\gamma_{1}^{(0)}, \gamma_{2}^{(0)}\right\}$ & $\sim \mathcal{E}_{\max }(1)$ & $\gamma_{\min }^{(i)} \sim \mathcal{E}(1)$ & $\gamma_{\max }^{(i)} \sim \mathcal{E}(1)$ & 1 & 1 & $2 \mathrm{rx}+\mathrm{asc}$ \\
\hline
\end{tabular}

RVs because the locations of the interfering BSs are the same but the power channel gains are related to different receive antennas. For all SWIPT schemes, nevertheless, the distribution of the aggregate other-cell interference is the same, i.e., $\Phi_{\mathcal{I}_{\mathrm{ID}}}(\cdot)=\Phi_{\mathcal{I}_{\mathrm{EH}}}(\cdot)=\Phi_{\mathcal{I}}(\cdot)$.

\section{A. Motivation: On the Benefits of Receiver Diversity}

To better motivate our research, let us compare PS and SAR schemes based on (4). From Table II and Remark 1, we evince that the power gains of the intended link and the aggregate other-cell interferences of both schemes are equivalent in distribution, e.g., they have the same PDF and CF. As far as the PS scheme is concerned, only a fraction of the received power is used by ID and EH units. As far as the SAR scheme is concerned, on the other hand, the entire received power is available at the input of both ID and EH units. As a result, $\mathcal{R}^{(\mathrm{SAR})} \geq \mathcal{R}^{(\mathrm{PS})}$ and $\mathcal{Q}^{(\mathrm{SAR})} \geq \mathcal{Q}^{(\mathrm{PS})}$ simultaneously, i.e., the SAR scheme always outperforms the PS scheme. This is obtained because $N_{r}=1$ and $N_{r}=2$ for PS and SAR schemes, respectively. From the implementation standpoint, the PS scheme requires appropriate circuits for splitting the received power while the SAR scheme avoids them by leveraging the availability of two receive antennas. This example shows the potential of using, whenever possible, multiple antennas at the LEDs. It highlights, in addition, the associated performance versus implementation trade-off, e.g., the possibility of replacing power splitters with an additional antenna element and radio frequency front-end. As far as the computational (signal processing) complexity of PS and SAR schemes is concerned, it is apparent that it is the same, since ID and EH receivers perform the same operations in both cases. 
Unlike the PS and SAR schemes, the comparison of the other schemes deserves more attention because of the different distribution of the channel power gain of the intended link, of the different power at the input of ID and EH units, and of the partial correlation of the aggregate other-cell interference. Quantifying the performance gain of each scheme compared to the others is, however, important because of the different implementation complexities, which are briefly summarized in Table II. Assessing these trade-offs is the ultimate objective of this paper.

\section{B. Problem Formulation}

As far as the LEDs as a whole are concerned, the trade-off between information rate and harvested power is quantified in terms of the J-CCDF of $\mathcal{R}$ and $\mathcal{Q}$ defined in (4) [18]:

$$
\bar{F}\left(\mathcal{R}_{*}, \mathcal{Q}_{*}\right)=\operatorname{Pr}\left\{\mathcal{R} \geq \mathcal{R}_{*}, \mathcal{Q} \geq \mathcal{Q}_{*}\right\}
$$

where $\mathcal{R}_{*}$ and $\mathcal{Q}_{*}$ are the minimum bit rate and harvested power, respectively, that are needed for the LEDs in order to be able to perform their tasks.

In the next sections, we compare the five SWIPT schemes introduced in Section II-E in terms of theirs J-CCDF and provide mathematical frameworks that allow us to optimize them and to decide the best scheme to use as a function of $\mathcal{R}_{*}$ and $\mathcal{Q}_{*}$.

\section{Performance Comparison and Expected Trends}

To facilitate the comparison among the SWIPT schemes, we start by introducing three remarks.

Remark 2: By using the Frechet inequality of the probability of logical conjunctions [20], the J-CCDF can be upper-bounded as follows:

$$
\bar{F}\left(\mathcal{R}_{*}, \mathcal{Q}_{*}\right) \leq \bar{F}_{\mathrm{UB}}\left(\mathcal{R}_{*}, \mathcal{Q}_{*}\right)=\min \left\{\operatorname{Pr}\left\{\mathcal{R} \geq \mathcal{R}_{*}\right\}, \operatorname{Pr}\left\{\mathcal{Q} \geq \mathcal{Q}_{*}\right\}\right\}
$$

By direct inspection, it is apparent that the upper-bound in (7) is asymptotically tight for every $\mathcal{Q}_{*}$ if $\mathcal{R}_{*} \rightarrow 0$ or $\mathcal{R}_{*} \rightarrow \infty$ and for every $\mathcal{R}_{*}$ if $\mathcal{Q}_{*} \rightarrow 0$ or $\mathcal{Q}_{*} \rightarrow \infty$. If $\mathcal{R}_{*} \ll 1$ or $\mathcal{Q}_{*} \gg 1$, the system operates in the EH-limited regime, i.e., $\bar{F}\left(\mathcal{R}_{*}, \mathcal{Q}_{*}\right) \approx \operatorname{Pr}\left\{\mathcal{Q} \geq \mathcal{Q}_{*}\right\}$. If $\mathcal{R}_{*} \gg 1$ or $\mathcal{Q}_{*} \ll 1$, the system operates in the ID-limited regime, i.e., $\bar{F}\left(\mathcal{R}_{*}, \mathcal{Q}_{*}\right) \approx \operatorname{Pr}\left\{\mathcal{R} \geq \mathcal{R}_{*}\right\}$. 
Remark 3: The J-CCDF of PS and PS-MRC schemes depends on $\rho$, i.e., $\operatorname{Pr}\left\{\mathcal{R} \geq \mathcal{R}_{*}\right\}$ $=\chi_{\mathrm{ID}}(\rho)$ and $\operatorname{Pr}\left\{\mathcal{Q} \geq \mathcal{Q}_{*}\right\}=\chi_{\mathrm{EH}}(\rho)$. In particular, $\chi_{\mathrm{ID}}(\cdot)$ and $\chi_{\mathrm{EH}}(\cdot)$ are monotonically decreasing and increasing functions of $\rho$, respectively, and, by definition, $\chi_{\mathrm{ID}}(0) \leq 1, \chi_{\mathrm{ID}}(1)=0$ and $\chi_{\mathrm{EH}}(0)=0, \chi_{\mathrm{EH}}(1) \leq 1$. Based on these properties and on the upper-bound in (7), we evince that an optimal value of $\rho, \rho_{\mathrm{opt}}$, that maximizes the J-CCDF exists and that it is the unique solution of the equation $\chi_{\mathrm{ID}}\left(\rho_{\mathrm{opt}}\right)=\chi_{\mathrm{EH}}\left(\rho_{\mathrm{opt}}\right)$. Since (7) is a upper-bound, however, the optimal value of $\rho$ that maximizes the exact J-CCDF may be different from the solution of the latter equation. The optimal power splitting ratios computed by using the exact J-CCDF and the upper-bound in (7) are compared in Section VI for some relevant case studies. Still based on (7), we evince that the J-CCDF satisfies the property that, a function of $\rho$, the equation $\chi(\rho)=\tau$, where $\chi(\rho)=\min \left\{\chi_{\mathrm{ID}}(\rho), \chi_{\mathrm{EH}}(\rho)\right\}$ and $0 \leq \tau \leq 1$ is a constant value, has at least one solution if $\tau \leq \chi\left(\rho_{\text {opt }}\right)$ and no solution if $\tau>\chi\left(\rho_{\text {opt }}\right)$.

Remark 4: Based on Remark 3, the upper-bound of the J-CCDF of PS and PS-MRC schemes attains its maximum if $\rho=\rho_{\text {opt }}$, where $\rho_{\text {opt }}$ is the solution of the equation $\chi_{\mathrm{ID}}\left(\rho_{\mathrm{opt}}\right)=\chi_{\mathrm{EH}}\left(\rho_{\mathrm{opt}}\right)$. Since $\chi_{\mathrm{ID}}(\cdot)$ and $\chi_{\mathrm{EH}}(\cdot)$ are monotonically decreasing and increasing functions of $\rho$, respectively, this implies that $\chi_{\mathrm{EH}}(\rho) \leq \chi_{\mathrm{ID}}(\rho)$ if $\rho \leq \rho_{\mathrm{opt}}$ and $\chi_{\mathrm{ID}}(\rho) \leq \chi_{\mathrm{EH}}(\rho)$ if $\rho \geq \rho_{\mathrm{opt}}$. Thus, the system operates in the EH-limited regime if $\rho \leq \rho_{\text {opt }}$, i.e., $\chi(\rho)=\min \left\{\chi_{\mathrm{ID}}(\rho), \chi_{\mathrm{EH}}(\rho)\right\}=\chi_{\mathrm{EH}}(\rho)$ and in the ID-limited regime if $\rho \geq \rho_{\mathrm{opt}}$, i.e., $\chi(\rho)=\min \left\{\chi_{\mathrm{ID}}(\rho), \chi_{\mathrm{EH}}(\rho)\right\}=\chi_{\mathrm{ID}}(\rho)$, respectively.

In Section III-A, we have shown that the SAR scheme always outperforms the PS scheme. In the next sub-sections, we explicitly compare the other SWIPT schemes. For ease of presentation, the following notation is used: $\bar{F}_{\mathrm{ID}}\left(\mathcal{R}_{*}\right)=\operatorname{Pr}\left\{\mathcal{R} \geq \mathcal{R}_{*}\right\}$ and $\bar{F}_{\mathrm{EH}}\left(\mathcal{Q}_{*}\right)=\operatorname{Pr}\left\{\mathcal{Q} \geq \mathcal{Q}_{*}\right\}$. Our derivations and conclusions are based on the upper-bound of the J-CCDF in (7).

\section{A. SAR versus ID-SC}

Lemma 1: Let us assume that $\mathcal{R}_{*}$ is given. The ID-SC scheme outperforms the SAR scheme, i.e., $\bar{F}_{\mathrm{UB}}^{(\mathrm{ID}-\mathrm{SC})}\left(\mathcal{R}_{*}, \mathcal{Q}_{*}\right) \geq \bar{F}_{\mathrm{UB}}^{(\mathrm{SAR})}\left(\mathcal{R}_{*}, \mathcal{Q}_{*}\right)$, if $\mathcal{Q}_{*}<\mathcal{Q}_{*}^{(c)}$, where $\mathcal{Q}_{*}^{(c)}$ is the unique solution of the equation $\bar{F}_{\mathrm{ID}}^{(\mathrm{SAR})}\left(\mathcal{R}_{*}\right)=\bar{F}_{\mathrm{EH}}^{(\mathrm{ID}-\mathrm{SC})}\left(\mathcal{Q}_{*}^{(c)}\right)$. On the other hand, the SAR scheme outperforms the ID-SC scheme if $\mathcal{Q}_{*}>\mathcal{Q}_{*}^{(c)}$. Let us assume that $\mathcal{Q}_{*}$ is given. The ID-SC scheme outperforms the 
SAR scheme if $\mathcal{R}_{*}>\mathcal{R}_{*}^{(c)}$, where $\mathcal{R}_{*}^{(c)}$ is the unique solution of the equation $\bar{F}_{\text {EH }}^{(\mathrm{ID}-\mathrm{SC})}\left(\mathcal{Q}_{*}\right)=$ $\bar{F}_{\mathrm{ID}}^{(\mathrm{SAR})}\left(\mathcal{R}_{*}^{(c)}\right)$. On the other hand, the SAR scheme outperforms the ID-SC scheme if $\mathcal{R}_{*}<\mathcal{R}_{*}^{(c)}$.

Proof: It is a direct consequence of the upper-bound in (7), by taking into account that: i) $\bar{F}_{\text {ID }}^{(\mathrm{ID}-\mathrm{SC})}\left(\mathcal{R}_{*}\right) \geq \bar{F}_{\mathrm{ID}}^{(\mathrm{SAR})}\left(\mathcal{R}_{*}\right)$ for every $\mathcal{R}_{*}$, since $\max \{a, b\} \geq a$ and $\max \{a, b\} \geq b$ for every $(a, b)$; ii) $\bar{F}_{\mathrm{EH}}^{(\mathrm{ID}-\mathrm{SC})}\left(\mathcal{Q}_{*}\right) \leq \bar{F}_{\mathrm{EH}}^{(\mathrm{SAR})}\left(\mathcal{Q}_{*}\right)$ for every $\mathcal{Q}_{*}$, since $\min \{a, b\} \leq a$ and $\min \{a, b\} \leq b$ for every $(a, b)$; iii) $\bar{F}_{\mathrm{ID}}(\cdot)$ is independent of $\mathcal{Q}_{*}$ and monotonically decreasing with $\mathcal{R}_{*}$; and iv) $\bar{F}_{\mathrm{EH}}(\cdot)$ is independent of $\mathcal{R}_{*}$ and monotonically decreasing with $\mathcal{Q}_{*}$.

From Lemma 1, we conclude that the ID-SC scheme outperforms the SAR scheme for large values of $\mathcal{R}_{*}$ and for small values of $\mathcal{Q}_{*}$, i.e., if the system operates in the ID-limited regime.

\section{B. SAR versus $E H-S C$}

Lemma 2: Let us assume that $\mathcal{R}_{*}$ is given. The EH-SC scheme outperforms the SAR scheme, i.e., $\bar{F}_{\mathrm{UB}}^{(\mathrm{EH}-\mathrm{SC})}\left(\mathcal{R}_{*}, \mathcal{Q}_{*}\right) \geq \bar{F}_{\mathrm{UB}}^{(\mathrm{SAR})}\left(\mathcal{R}_{*}, \mathcal{Q}_{*}\right)$, if $\mathcal{Q}_{*}>\mathcal{Q}_{*}^{(c)}$, where $\mathcal{Q}_{*}^{(c)}$ is the unique solution of the equation $\bar{F}_{\mathrm{ID}}^{(\mathrm{EH}-\mathrm{SC})}\left(\mathcal{R}_{*}\right)=\bar{F}_{\mathrm{EH}}^{(\mathrm{SAR})}\left(\mathcal{Q}_{*}^{(c)}\right)$. On the other hand, the SAR scheme outperforms the EH-SC scheme if $\mathcal{Q}_{*}<\mathcal{Q}_{*}^{(c)}$. Let us assume that $\mathcal{Q}_{*}$ is given. the EH-SC scheme outperforms the SAR scheme if $\mathcal{R}_{*}<\mathcal{R}_{*}^{(c)}$, where $\mathcal{R}_{*}^{(c)}$ is the unique solution of the equation $\bar{F}_{\mathrm{EH}}^{(\mathrm{SAR})}\left(\mathcal{Q}_{*}\right)=$ $\bar{F}_{\mathrm{ID}}^{(\mathrm{EH}-\mathrm{SC})}\left(\mathcal{R}_{*}^{(c)}\right)$. On the other hand, the SAR scheme outperforms the EH-SC scheme if $\mathcal{R}_{*}>$ $\mathcal{R}_{*}^{(c)}$.

Proof: It follows from the upper-bound in (7), by using the same line of thought as that of the proof of Lemma 1. For brevity, the details of the derivation are omitted.

From Lemma 2, we conclude that the EH-SC scheme outperforms the SAR scheme for large values of $\mathcal{Q}_{*}$ and for small values of $\mathcal{R}_{*}$, i.e., if the system operates in the EH-limited regime.

\section{ID-SC versus EH-SC}

Lemma 3: Let us assume that $\mathcal{R}_{*}$ is given. The ID-SC scheme outperforms the EH-SC scheme, i.e., $\bar{F}_{\mathrm{UB}}^{(\mathrm{ID}-\mathrm{SC})}\left(\mathcal{R}_{*}, \mathcal{Q}_{*}\right) \geq \bar{F}_{\mathrm{UB}}^{(\mathrm{EH}-\mathrm{SC})}\left(\mathcal{R}_{*}, \mathcal{Q}_{*}\right)$, if $\mathcal{Q}_{*}<\mathcal{Q}_{*}^{(c)}$, where $\mathcal{Q}_{*}^{(c)}$ is the unique solution of the equation $\bar{F}_{\mathrm{ID}}^{(\mathrm{EH}-\mathrm{SC})}\left(\mathcal{R}_{*}\right)=\bar{F}_{\mathrm{EH}}^{(\mathrm{ID}-\mathrm{SC})}\left(\mathcal{Q}_{*}^{(c)}\right)$. On the other hand, the EH-SC scheme outperforms the ID-SC scheme if $\mathcal{Q}_{*}>\mathcal{Q}_{*}^{(c)}$. Let us assume that $\mathcal{Q}_{*}$ is given. The ID-SC scheme outperforms the EH-SC scheme if $\mathcal{R}_{*}>\mathcal{R}_{*}^{(c)}$, where $\mathcal{R}_{*}^{(c)}$ is the unique solution of the 
equation $\bar{F}_{\mathrm{EH}}^{(\mathrm{ID}-\mathrm{SC})}\left(\mathcal{Q}_{*}\right)=\bar{F}_{\mathrm{ID}}^{(\mathrm{EH}-\mathrm{SC})}\left(\mathcal{R}_{*}^{(c)}\right)$. On the other hand, the EH-SC scheme outperforms the ID-SC scheme if $\mathcal{R}_{*}<\mathcal{R}_{*}^{(c)}$.

Proof: It follows from the upper-bound in (7), by using the same line of thought as that of the proof of Lemma 1 and by noting that $\max \{a, b\} \geq \min \{a, b\}$ for every $(a, b)$.

From Lemma 3, we conclude that the ID-SC scheme is to be preferred to the EH-SC scheme if the system operates in the ID-limited regime. If the system operates in the EH-limited regime, on the other hand, the EH-SC scheme is to be preferred to the ID-SC scheme.

Remark 5: From Lemmas 1-3, three main conclusions can be drawn: 1) there is no SWIPT scheme among the SAR, ID-SC and EH-SC schemes that outperforms the others for every pair $\left.\left(\mathcal{R}_{*}, \mathcal{Q}_{*}\right) ; 2\right)$ the SAR scheme is a special case of a generalized SWIPT scheme that is obtained by choosing, as a function of $\left(\mathcal{R}_{*}, \mathcal{Q}_{*}\right)$, the best scheme between the ID-SC and the EH-SC schemes; and 3) given the reliability constraints $\left(\mathcal{R}_{*}, \mathcal{Q}_{*}\right)$, the performance of SWIPT-enabled cellular networks can be optimized by enabling the LEDs to adaptively use, as a function of $\left(\mathcal{R}_{*}, \mathcal{Q}_{*}\right)$, either the ID-SC scheme or the EH-SC scheme.

Based on Remark 5, we introduce a new SWIPT scheme that is referred to as Adaptive Selection Combining (A-SC), which subsumes SAR, ID-SC and EH-SC schemes and foresees that the LEDs use the best SWIPT scheme, between ID-SC and EH-SC, as a function of $\left(\mathcal{R}_{*}, \mathcal{Q}_{*}\right)$. More specifically, the LEDs operate by using either the ID-SC or the EH-SC scheme according to the switching points $\mathcal{Q}_{*}^{(c)}$ and $\mathcal{R}_{*}^{(c)}$ introduced in Lemmas 1-3. The practical implementation of this adaptive scheme is elaborated at the end of Section IV-F.

\section{PS-MRC versus A-SC}

Lemma 4: Let us assume that the PS-MRC scheme operates at its optimum $\rho_{\text {opt }}$, which is obtained by maximizing its J-CCDF as a function of $\rho$. The PS-MRC scheme outperforms the A-SC scheme if they both operate either in the ID-limited regime or in the EH-limited regime.

Proof: In the ID-limited regime and in the EH-limited regime, we have $\bar{F}_{\mathrm{UB}}^{(\mathrm{PS}-\mathrm{MRC})}\left(\mathcal{R}_{*}, \mathcal{Q}_{*}\right) \approx$ $\bar{F}_{\mathrm{ID}}^{(\mathrm{PS}-\mathrm{MRC})}\left(\mathcal{R}_{*}\right)$ and $\bar{F}_{\mathrm{UB}}^{(\mathrm{PS}-\mathrm{MRC})}\left(\mathcal{R}_{*}, \mathcal{Q}_{*}\right) \approx \bar{F}_{\mathrm{EH}}^{(\mathrm{PS}-\mathrm{MRC})}\left(\mathcal{Q}_{*}\right)$, respectively. This implies that $\rho_{\mathrm{opt}}=0$ and $\rho_{\mathrm{opt}}=1$ in the ID-limited regime and in the EH-limited regime, respectively. In addition, the A-SC scheme reduces to the ID-SC scheme and to the EH-SC scheme in the 
ID-limited regime and in the EH-limited regime, respectively. The proof follows from the upperbound in (7), by noting that $a+b \geq \max \{a, b\}$ for every non-negative $(a, b)$.

Lemma 5: Let us assume that the PS-MRC scheme operates at its optimum $\rho_{\text {opt }}$, which is obtained by maximizing its J-CCDF as a function of $\rho$. For a given pair $\left(\mathcal{R}_{*}, \mathcal{Q}_{*}\right)$, the PS-MRC scheme outperforms the A-SC scheme if, as a function of $\rho$, the equation $\bar{F}_{\mathrm{UB}}^{\text {(PS-MRC) }}\left(\mathcal{R}_{*}, \mathcal{Q}_{*} ; \rho\right)=$ $\bar{F}_{\mathrm{UB}}^{(\mathrm{A}-\mathrm{SC})}\left(\mathcal{R}_{*}, \mathcal{Q}_{*}\right)$ admits at least one solution for $0 \leq \rho \leq 1$. If the equation admits no solution, on the other hand, the A-SC scheme outperforms the PS-MRC scheme.

Proof: It follows by applying the upper-bound in (7) and from Remark 3.

\section{E. $P S-M R C$ versus $P S$}

Lemma 6: Let us assume that the PS-MRC scheme and the PS scheme operate by using the same $\rho$. The PS-MRC scheme outperforms the PS scheme for every $\left(\mathcal{R}_{*}, \mathcal{Q}_{*}\right)$.

Let us assume, on the other hand, that the PS-MRC scheme and the PS scheme operate at their respective optima $\rho_{\mathrm{opt}}^{(\mathrm{PS}-\mathrm{MRC})}$ and $\rho_{\mathrm{opt}}^{(\mathrm{PS})}$, which are obtained by finding the maximum, as a function of $\rho$, of $\bar{F}_{\mathrm{UB}}^{(\mathrm{PS}-\mathrm{MRC})}\left(\mathcal{R}_{*}, \mathcal{Q}_{*} ; \rho\right)$ and $\bar{F}_{\mathrm{UB}}^{(\mathrm{PS})}\left(\mathcal{R}_{*}, \mathcal{Q}_{*} ; \rho\right)$, respectively. The PS-MRC scheme outperforms the PS scheme for every $\left(\mathcal{R}_{*}, \mathcal{Q}_{*}\right)$.

Proof: Let us assume that $\left(\mathcal{R}_{*}, \mathcal{Q}_{*}\right)$ is given. Let us use the same notation as in Remark 3, i.e., $\bar{F}^{(\mathrm{PS}-\mathrm{MRC})}\left(\mathcal{R}_{*}, \mathcal{Q}_{*} ; \rho\right) \leq \chi^{(\mathrm{PS}-\mathrm{MRC})}(\rho)=\min \left\{\chi_{\mathrm{ID}}^{(\mathrm{PS}-\mathrm{MRC})}(\rho), \chi_{\mathrm{EH}}^{(\mathrm{PS}-\mathrm{MRC})}(\rho)\right\}$ and $\bar{F}^{(\mathrm{PS})}\left(\mathcal{R}_{*}, \mathcal{Q}_{*} ; \rho\right) \leq \chi^{(\mathrm{PS})}(\rho)=\min \left\{\chi_{\mathrm{ID}}^{(\mathrm{PS})}(\rho), \chi_{\mathrm{EH}}^{(\mathrm{PS})}(\rho)\right\}$. Since $a+b \geq a$ and $a+b \geq b$ for every non-negative $(a, b)$, we obtain $\chi_{\mathrm{ID}}^{(\mathrm{PS}-\mathrm{MRC})}(\rho) \geq \chi_{\mathrm{ID}}^{(\mathrm{PS})}(\rho)$ and $\chi_{\mathrm{EH}}^{(\mathrm{PS}-\mathrm{MRC})}(\rho) \geq \chi_{\mathrm{EH}}^{(\mathrm{PS})}(\rho)$ for every $0 \leq \rho \leq 1$. This proves the first part.

Based on the upper-bound in (7) and on Remark 3, $\rho_{\mathrm{opt}}^{(\mathrm{PS}-\mathrm{MRC})}$ and $\rho_{\mathrm{opt}}^{(\mathrm{PS})}$ satisfy the equalities $\chi_{\mathrm{ID}}^{(\mathrm{PS}-\mathrm{MRC})}\left(\rho_{\mathrm{opt}}^{(\mathrm{PS}-\mathrm{MRC})}\right)=\chi_{\mathrm{EH}}^{(\mathrm{PS}-\mathrm{MRC})}\left(\rho_{\mathrm{opt}}^{(\mathrm{PS}-\mathrm{MRC})}\right)$ and $\chi_{\mathrm{ID}}^{(\mathrm{PS})}\left(\rho_{\mathrm{opt}}^{(\mathrm{PS})}\right)=\chi_{\mathrm{EH}}^{(\mathrm{PS})}\left(\rho_{\mathrm{opt}}^{(\mathrm{PS})}\right)$, respectively. Accordingly, the following holds:

$$
\begin{array}{r}
\bar{F}^{(\mathrm{PS}-\mathrm{MRC})}\left(\mathcal{R}_{*}, \mathcal{Q}_{*} ; \rho_{\mathrm{opt}}^{(\mathrm{PS}-\mathrm{MRC})}\right) \leq \chi^{(\mathrm{PS}-\mathrm{MRC})}\left(\rho_{\mathrm{opt}}^{(\mathrm{PS}-\mathrm{MRC})}\right) \stackrel{(a)}{=} \chi_{\mathrm{EH}}^{(\mathrm{PS}-\mathrm{MRC})}\left(\rho_{\mathrm{opt}}^{(\mathrm{PS}-\mathrm{MRC})}\right) \\
\stackrel{(b)}{\geq} \chi_{\mathrm{EH}}^{(\mathrm{PS}-\mathrm{MRC})}(\rho) \stackrel{(c)}{\geq} \chi_{\mathrm{EH}}^{(\mathrm{PS})}(\rho) \stackrel{(d)}{=} \chi^{(\mathrm{PS})}(\rho) \geq \bar{F}^{(\mathrm{PS})}\left(\mathcal{R}_{*}, \mathcal{Q}_{*} ; \rho\right),
\end{array}
$$

where (a) follows from $\chi_{\mathrm{ID}}^{(\mathrm{PS}-\mathrm{MRC})}\left(\rho_{\mathrm{opt}}^{(\mathrm{PS}-\mathrm{MRC})}\right)=\chi_{\mathrm{EH}}^{(\mathrm{PS}-\mathrm{MRC})}\left(\rho_{\mathrm{opt}}^{(\mathrm{PS}-\mathrm{MRC})}\right)$, (b) holds for $\rho \leq$ $\rho_{\mathrm{opt}}^{(\mathrm{PS}-\mathrm{MRC})}$ since $\chi_{\mathrm{EH}}(\cdot)$ is a monotonically increasing function of $\rho$, (c) follows from the first 
part of the proof, and (d) holds for $\rho \leq \rho_{\mathrm{opt}}^{(\mathrm{PS})}$ since $\chi_{\mathrm{EH}}^{(\mathrm{PS})}(\rho) \leq \chi_{\mathrm{ID}}^{(\mathrm{PS})}(\rho)$ if $\rho \leq \rho_{\mathrm{opt}}^{(\mathrm{PS})}$ (see Remark 4 for the details). By using a similar line of thought, the following holds:

$$
\begin{array}{r}
\bar{F}^{(\mathrm{PS}-\mathrm{MRC})}\left(\mathcal{R}_{*}, \mathcal{Q}_{*} ; \rho_{\mathrm{opt}}^{(\mathrm{PS}-\mathrm{MRC})}\right) \leq \chi^{(\mathrm{PS}-\mathrm{MRC})}\left(\rho_{\mathrm{opt}}^{(\mathrm{PS}-\mathrm{MRC})}\right)=\chi_{\mathrm{ID}}^{(\mathrm{PS}-\mathrm{MRC})}\left(\rho_{\mathrm{opt}}^{(\mathrm{PS}-\mathrm{MRC})}\right) \\
\geq \chi_{\mathrm{ID}}^{(\mathrm{PS}-\mathrm{MRC})}(\rho) \geq \chi_{\mathrm{ID}}^{(\mathrm{PS})}(\rho)=\chi^{(\mathrm{PS})}(\rho) \geq \bar{F}^{(\mathrm{PS})}\left(\mathcal{R}_{*}, \mathcal{Q}_{*} ; \rho\right)
\end{array}
$$

for $\rho \geq \rho_{\mathrm{opt}}^{(\mathrm{PS}-\mathrm{MRC})}$ and $\rho \geq \rho_{\mathrm{opt}}^{(\mathrm{PS})}$.

From (8) and (9), in conclusion, we obtain $\bar{F}^{(\mathrm{PS}-\mathrm{MRC})}\left(\mathcal{R}_{*}, \mathcal{Q}_{*} ; \rho_{\mathrm{opt}}^{(\mathrm{PS}-\mathrm{MRC})}\right) \geq \bar{F}^{(\mathrm{PS})}\left(\mathcal{R}_{*}, \mathcal{Q}_{*} ; \rho\right)$

if $\rho \leq \min \left\{\rho_{\mathrm{opt}}^{(\mathrm{PS}-\mathrm{MRC})}, \rho_{\mathrm{opt}}^{(\mathrm{PS})}\right\}$ or $\rho \geq \max \left\{\rho_{\mathrm{opt}}^{(\mathrm{PS}-\mathrm{MRC})}, \rho_{\mathrm{opt}}^{(\mathrm{PS})}\right\}$. This implies that, for every $\left(\rho_{\mathrm{opt}}^{\text {(PS-MRC) }}, \rho_{\mathrm{opt}}^{(\mathrm{PS})}\right)$ and $\left(\mathcal{R}_{*}, \mathcal{Q}_{*}\right)$, the PS-MRC scheme outperforms the PS scheme if they operate at their respective optima as a function of $\rho$. This concludes the proof.

Lemma 6 establishes that PS-MRC is superior to PS if either they use the same power splitting ratio or they operate at their respective optimal power splitting ratios. For arbitrary values of their power splitting ratios, on the other hand, no general conclusion can be drawn. Numerical examples are illustrated in Section VI for some relevant case studies.

\section{F. Main Performance Trends}

From the comparative analysis of the SWIPT schemes, three main conclusions can be drawn.

1) There exists a unique value of $\rho$ that optimizes the performance of PS and PS-MRC schemes. PS and PS-MRC schemes that operate at their respective optima are referred to as Optimum PS (OPS) and Optimum PS-MRC (OPS-MRC) schemes, respectively.

2) For every pair $\left(\mathcal{R}_{*}, \mathcal{Q}_{*}\right)$, i) the A-SC scheme outperforms the SAR scheme, ii) the SAR scheme outperforms the PS scheme, iii) the PS-MRC scheme outperforms the PS scheme if $\rho$ is the same, iv) and the OPS-MRC scheme outperforms the OPS scheme. This implies that the A-SC scheme outperforms the PS scheme.

3) The OPS-MRC scheme outperforms or underperforms the A-SC scheme depending on the pair $\left(\mathcal{R}_{*}, \mathcal{Q}_{*}\right)$ and on the specific setup of parameters being considered (see Lemma 5).

In order to optimize the J-CCDF, our analysis suggests that the LEDs need to operate in an adaptive fashion by choosing the SWIPT scheme to use as a function of $\left(\mathcal{R}_{*}, \mathcal{Q}_{*}\right)$. Depending on the application, in general, each LED may have different $\left(\mathcal{R}_{*}, \mathcal{Q}_{*}\right)$ requirements. Concretely, an 
adaptive SWIPT scheme can be implemented as follows. Assume that mathematical frameworks for the J-CCDF of ID-SC, EH-SC and PS-MRC schemes are available in a tractable and computable form. For any $\left(\mathcal{R}_{*}, \mathcal{Q}_{*}\right)$ of interest, either the BSs or the LEDs (depending on their computational complexity capabilities) adaptively choose the best SWIPT scheme to use as follows: 1) the power splitting coefficient that optimizes the J-CCDF of the PS-MRC scheme is estimated and the related optimal J-CCDF is computed, 2) the J-CCDF of the ID-SC and EHSC schemes are computed, 3) the LEDs use the SWIPT scheme that provides the best J-CCDF among the three. If the BSs perform these tasks, they need to forward the related information to their intended LEDs. This usually requires just a few control bits (two in the considered setup). A system that operates according to this adaptive policy is referred to as Adaptive SWIPT $(A-S W I P T)$. To implement the A-SWIPT scheme in practice, mathematical expressions of the J-CCDF of all SWIPT schemes studied in this paper are provided in the next section.

It is worth emphasizing that, by definition, the J-CCDF in (6) is obtained by computing the average with respect to the spatial topology of the cellular network and with respect to the channel conditions. This implies that the considered (system-level) optimization can be performed off-line with a minimum overhead, since it depends on system parameters that are slowly-varying, e.g., that usually change on a geographical scale. For example, it can be performed at the beginning of each communication round and if the performance requirements $\left(\mathcal{R}_{*}, \mathcal{Q}_{*}\right)$ change.

\section{System-LEVEl AnAlysis}

The following two propositions provide mathematical expressions of the J-CCDF for the five SWIPT schemes introduced in Section II-E. Proposition 1 is exact and is applicable only to PS and PS-MRC schemes. Proposition 2 is based on the upper-bound in (7) and is applicable to all SWIPT schemes. An exact mathematical framework for SAR, ID-SC and EH-SC schemes may be obtained by using the multi-dimensional inversion theorem in [21]. The resulting framework, however, would be formulated in terms of multi-fold integrals with limited mathematical and numerical tractability. For this reason, it is not explicitly considered in this paper. For the interested readers, however, some hints on how to exploit the multi-dimensional inversion theorem for

computing the J-CCDF are reported in Appendix A. The mathematical complexity of studying 
SAR, ID-SC and EH-SC schemes compared with PS and PS-MRC schemes originates from the fact that the aggregate other-cell interferences of ID and EH units are dissimilar and are only equivalent in distribution (see Remark 1).

Proposition 1: Let $f_{L^{(0)}}(\cdot)$ be the PDF of the smallest path-loss, $L^{(0)}$, defined in (2):

$$
f_{L^{(0)}}(x)=\widehat{\Lambda}([0, x)) \exp (-\Lambda([0, x)))
$$

where $\Lambda([0, x))=\Lambda_{\mathrm{LOS}}([0, x))+\Lambda_{\mathrm{NLOS}}([0, x))$ is the intensity measure of the PPP of the path-losses [18], $\widehat{\Lambda}([0, x))=\widehat{\Lambda}_{\mathrm{LOS}}([0, x))+\widehat{\Lambda}_{\mathrm{NLOS}}([0, x))$ is its first derivative computed with respect to $x$, as well as, for $s \in\{\operatorname{LOS}, \operatorname{NLOS}\}, \Lambda_{s}([\cdot, \cdot))$ and $\widehat{\Lambda}_{s}([\cdot, \cdot))$ are the intensity measure and its first derivative, respectively, of the PPP of the path-losses in state $s$ defined as follows:

$$
\begin{aligned}
\Lambda_{s}([0, x)) & =\pi \lambda q_{s}^{[0, D]}\left(\frac{x}{\kappa_{0}}\right)^{\frac{2}{\beta_{s}}} \overline{\mathcal{H}}\left(x-\kappa_{0} D^{\beta_{s}}\right) \\
& +\pi \lambda\left(\left(\frac{x}{\kappa_{0}}\right)^{\frac{2}{\beta_{s}}} q_{s}^{[D, \infty]}+D^{2}\left(q_{s}^{[0, D]}-q_{s}^{[D, \infty]}\right)\right) \mathcal{H}\left(x-\kappa_{0} D^{\beta_{s}}\right), \\
\widehat{\Lambda}_{s}([0, x)) & =\left(2 \pi \lambda / \beta_{s}\right) q_{s}^{[0, D]} \kappa_{0}^{-2 / \beta_{s}} x^{\left(2 / \beta_{s}-1\right)} \overline{\mathcal{H}}\left(x-\kappa_{0} D^{\beta_{s}}\right) \\
& +\left(2 \pi \lambda / \beta_{s}\right) q_{s}^{[D, \infty]} \kappa_{0}^{-2 / \beta_{s}} x^{\left(2 / \beta_{s}-1\right)} \mathcal{H}\left(x-\kappa_{0} D^{\beta_{s}}\right) .
\end{aligned}
$$

Let $\Phi_{\mathcal{I}}\left(\cdot \mid L^{(0)}\right)$ be the CF of the other-cell interference, $\mathcal{I}$, conditioned on $L^{(0)}$ given in (5):

$$
\Phi_{\mathcal{I}}\left(\omega \mid L^{(0)}\right)=\Phi_{\mathcal{I}}\left(\omega \mid L^{(0)} ; \mathrm{LOS}\right) \Phi_{\mathcal{I}}\left(\omega \mid L^{(0)} ; \mathrm{NLOS}\right)
$$

where, for $s \in\{\operatorname{LOS}, \mathrm{NLOS}\}, \Phi_{\mathcal{I}}\left(\cdot \mid L^{(0)} ; s\right)$ is, conditioned on $L^{(0)}$, the CF of the other-cell interference of all links in state $s$ defined as follows:

$$
\begin{aligned}
& \Phi_{\mathcal{I}}\left(\omega \mid L^{(0)} ; s\right)=\exp \left(\lambda \pi q_{s}^{[D, \infty]} \max \left\{D^{2},\left(L^{(0)} / \kappa_{0}\right)^{2 / \beta_{s}}\right\}\left(1-\Upsilon_{s}\left(\omega, \max \left\{\kappa_{0} D^{\beta_{s}}, L^{(0)}\right\}\right)\right)\right) \\
& \times \exp \left(\pi \lambda q_{s}^{[0, D]}\left[\left(L^{(0)} / \kappa_{0}\right)^{2 / \beta_{s}}\left(1-\Upsilon_{s}\left(\omega, L^{(0)}\right)\right)-D^{2}\left(1-\Upsilon_{s}\left(\omega, \kappa_{0} D^{\beta_{s}}\right)\right)\right] \overline{\mathcal{H}}\left(L^{(0)}-\kappa_{0} D^{\beta_{s}}\right)\right),
\end{aligned}
$$

and $\Upsilon_{s}(\cdot, \cdot)$ is the following short-hand:

$$
\Upsilon_{s}(\omega, \mathrm{Z})=\frac{\theta_{\mathrm{M}}}{2 \pi}{ }_{2} F_{1}\left(1,-\frac{2}{\beta_{s}}, 1-\frac{2}{\beta_{s}}, \frac{j \omega}{Z} G_{\mathrm{M}}\right)+\left(1-\frac{\theta_{\mathrm{M}}}{2 \pi}\right){ }_{2} F_{1}\left(1,-\frac{2}{\beta_{s}}, 1-\frac{2}{\beta_{s}}, \frac{j \omega}{Z} G_{\mathrm{S}}\right) .
$$


TABLE III: Definitions of $\left(a_{\mathrm{ID}}, b_{\mathrm{ID}}, c_{\mathrm{ID}}\right)$ and $\left(a_{\mathrm{EH}}, b_{\mathrm{EH}}, c_{\mathrm{EH}}\right)$ according to the PDFs in Table II.

\begin{tabular}{|c||c|c|c||c|c|c|}
\hline & $a_{\mathrm{ID}}$ & $b_{\mathrm{ID}}$ & $c_{\mathrm{ID}}$ & $a_{\mathrm{EH}}$ & $b_{\mathrm{EH}}$ & $c_{\mathrm{EH}}$ \\
\hline \hline PS & 1 & 0 & 0 & 1 & 0 & 0 \\
\hline PS-MRC & 0 & 0 & 1 & 0 & 0 & 1 \\
\hline SAR & 1 & 0 & 0 & 1 & 0 & 0 \\
\hline ID-SC & 2 & -2 & 0 & 0 & 2 & 0 \\
\hline EH-SC & 0 & 2 & 0 & 2 & -2 & 0 \\
\hline
\end{tabular}

Then, the J-CCDF of PS and PS-MRC schemes can be formulated as follows:

$$
\bar{F}\left(\mathcal{R}_{*}, \mathcal{Q}_{*}\right)=\int_{0}^{+\infty} \int_{0}^{+\infty} \frac{1}{\pi \omega} \operatorname{Im}\left\{\mathcal{J}(\omega, y) \Phi_{\mathcal{I}}\left(\frac{\omega}{G^{(0)}} \mid y\right)\right\} f_{L^{(0)}}(y) d \omega d y
$$

where $u=0$ for PS and $u=1$ for PS-MRC schemes, respectively, and, for notational simplicity, the following short-hand is introduced:

$$
\begin{aligned}
\mathcal{J}(\omega, y) & =\exp \left(-j \omega \frac{q_{*}}{P G^{(0)}}\right)\left(1-\frac{j \omega}{y}\right)^{-(1+u)} \Gamma\left(1+u, \frac{\mathcal{T}_{*}}{P G^{(0)}}(y-j \omega)\right) \\
& -\exp \left(j \omega \frac{\sigma_{*}^{2}}{P G^{(0)}}\right)\left(1+\frac{j \omega r_{*}}{y}\right)^{-(1+u)} \Gamma\left(1+u, \frac{\mathcal{T}_{*}}{P G^{(0)}}\left(y+j \omega r_{*}\right)\right) .
\end{aligned}
$$

Proof: Since $\mathcal{U}_{\mathrm{ID}}^{(0)}=\mathcal{U}_{\mathrm{EH}}^{(0)}=\mathcal{U}^{(0)}$ for PS and PS-MRC schemes (see Table II), from (4) the $\mathrm{J}-\mathrm{CCDF}$ can be formulated as follows:

$$
\begin{aligned}
\bar{F}\left(\mathcal{R}_{*}, \mathcal{Q}_{*}\right) & =\operatorname{Pr}\left\{\mathcal{R} \geq \mathcal{R}_{*}, \mathcal{Q} \geq \mathcal{Q}_{*}\right\}=\operatorname{Pr}\left\{\mathcal{I} \leq G^{(0)} \mathcal{U}^{(0)} r_{*} / L^{(0)}-\sigma_{*}^{2} / P, \mathcal{I} \geq-G^{(0)} \mathcal{U}^{(0)} / L^{(0)}+q_{*} / P\right\} \\
& = \begin{cases}\operatorname{Pr}\left\{-G^{(0)} \mathcal{U}^{(0)} / L^{(0)}+q_{*} / P \leq \mathcal{I} \leq G^{(0)} \mathcal{U}^{(0)} r_{*} / L^{(0)}-\sigma_{*}^{2} / P\right\} & \text { if } G^{(0)} \mathcal{U}^{(0)} \geq\left(\mathcal{T}_{*} / P\right) L^{(0)} \\
0 & \text { otherwise }\end{cases} \\
& =\mathbb{E}_{L^{(0)}}\left\{\int_{\left(\mathcal{T}_{*} / P\right)\left(L^{(0)} / G^{(0)}\right)}^{+\infty} F_{\mathcal{I}}\left(G^{(0)} x r_{*} / L^{(0)}-\sigma_{*}^{2} / P \mid L^{(0)}\right) f_{\mathcal{U}^{(0)}}(x) d x\right\} \\
& -\mathbb{E}_{L^{(0)}}\left\{\int_{\left(\mathcal{T}_{*} / P\right)\left(L^{(0)} / G^{(0)}\right)}^{+\infty} F_{\mathcal{I}}\left(-G^{(0)} x / L^{(0)}+q_{*} / P \mid L^{(0)}\right) f_{\mathcal{U}^{(0)}}(x) d x\right\}
\end{aligned}
$$

where $F_{\mathcal{I}}(x)=\operatorname{Pr}\{\mathcal{I}<x\}$ is the CDF of $\mathcal{I}$. With the aid of the Gil-Pelaez theorem [22, Eq. (16)], $F_{\mathcal{I}}(\cdot)$ can be formulated in terms of the $\mathrm{CF}$ of $\mathcal{I}$. The proof follows by computing the PDF of $L^{(0)}$ and the CF of $\mathcal{I}$ as in [18, Lemma 1] and [18, Lemma 2], respectively, by inserting them in (18) and by using some algebra.

Proposition 2: Let $f_{L^{(0)}}(\cdot)$ be the PDF in (10) and $\Phi_{\mathcal{I}}(\cdot \mid \cdot)$ be the CF in (13). Let $\widehat{\mathcal{M}}_{\mathcal{I}}(z)=$ $d \mathcal{M}_{\mathcal{I}}(z) / d z$ be the first derivative of the MGF of the aggregate other-cell interference, i.e., 
$\mathcal{M}_{\mathcal{I}}(z)=\Phi_{\mathcal{I}}(-j z \mid z)$. Then, the J-CCDF of the SWIPT schemes in Table II can be upperbounded as $\bar{F}\left(\mathcal{R}_{*}, \mathcal{Q}_{*}\right) \leq \min \left\{\bar{F}_{\text {ID }}\left(\mathcal{R}_{*}\right), \bar{F}_{\text {EH }}\left(\mathcal{Q}_{*}\right)\right\}$, where $\bar{F}_{\text {ID }}\left(\mathcal{R}_{*}\right)=\operatorname{Pr}\left\{\mathcal{R} \geq \mathcal{R}_{*}\right\}$ and $\bar{F}_{\mathrm{EH}}\left(\mathcal{Q}_{*}\right)=\operatorname{Pr}\left\{\mathcal{Q} \geq \mathcal{Q}_{*}\right\}$ can be formulated, respectively, as follows:

$$
\begin{gathered}
\bar{F}_{\mathrm{ID}}\left(\mathcal{R}_{*}\right)=\int_{0}^{+\infty} \mathcal{J}_{\mathrm{ID}}(y) f_{L^{(0)}}(y) d y \\
\bar{F}_{\mathrm{EH}}\left(\mathcal{Q}_{*}\right)=\frac{1}{2}+\int_{0}^{+\infty} \int_{0}^{+\infty} \frac{1}{\pi \omega} \operatorname{Im}\left\{\exp \left(-j \omega \frac{\tilde{q}_{*}}{P}\right) \mathcal{J}_{\mathrm{EH}}\left(\omega, \frac{G^{(0)}}{y}\right) \Phi_{\mathcal{I}}(\omega \mid y)\right\} f_{L^{(0)}}(y) d \omega d y
\end{gathered}
$$

where, for ease of notation, the following short-hands are introduced:

$$
\begin{aligned}
\mathcal{J}_{\mathrm{ID}}(y)= & \left(a_{\mathrm{ID}}+c_{\mathrm{ID}}\right) \exp \left(-\frac{\tilde{\sigma}_{*}^{2} y}{P G^{(0)} \tilde{r}_{*}}\right) \mathcal{M}_{\mathcal{I}}\left(\frac{y}{G^{(0)} \tilde{r}_{*}}\right) \\
& +\frac{b_{\mathrm{ID}}}{2} \exp \left(-\frac{2 \tilde{\sigma}_{*}^{2} y}{P G^{(0)} \tilde{r}_{*}}\right) \mathcal{M}_{\mathcal{I}}\left(\frac{2 y}{G^{(0)} \tilde{r}_{*}}\right) \\
& +c_{\mathrm{ID}} \frac{\tilde{\sigma}_{*}^{2} y}{P G^{(0)} \tilde{r}_{*}} \exp \left(-\frac{\tilde{\sigma}_{*}^{2} y}{P G^{(0)} \tilde{r}_{*}}\right) \mathcal{M}_{\mathcal{I}}\left(\frac{y}{G^{(0)} \tilde{r}_{*}}\right) \\
& -c_{\mathrm{ID}} \frac{y}{G^{(0)} \tilde{r}_{*}} \exp \left(-\frac{\tilde{\sigma}_{*}^{2} y}{P G^{(0)} \tilde{r}_{*}}\right) \widehat{\mathcal{M}}_{\mathcal{I}}\left(\frac{y}{G^{(0)} \tilde{r}_{*}}\right), \\
\mathcal{J}_{\mathrm{EH}}(\omega, z)= & a_{\mathrm{EH}}(1-j \omega z)^{-1}+b_{\mathrm{EH}}(2-j \omega z)^{-1}+c_{\mathrm{EH}}(1-j \omega z)^{-2},
\end{aligned}
$$

and the two triplets of coefficients $\left(a_{\mathrm{ID}}, b_{\mathrm{ID}}, c_{\mathrm{ID}}\right)$ and $\left(a_{\mathrm{EH}}, b_{\mathrm{EH}}, c_{\mathrm{EH}}\right)$ are defined in Table III.

Proof: $\bar{F}_{\mathrm{ID}}(\cdot)$ is obtained by using the Pcov-based approach in $\left[6\right.$, Sec. III-G] and $\bar{F}_{\mathrm{ID}}(\cdot)$ is obtained by using the Gil-Pelaez theorem in [22]. The triplets $\left(a_{\mathrm{ID}}, b_{\mathrm{ID}}, c_{\mathrm{ID}}\right)$ and $\left(a_{\mathrm{EH}}, b_{\mathrm{EH}}, c_{\mathrm{EH}}\right)$ are obtained based on the PDFs in Table II. The proof follows with the aid of some algebra.

The J-CCDFs in Proposition 1 and Proposition 2 are formulated in terms of two-fold integrals that can be efficiently computed with the aid of state-of-the-art computational software programs and have the advantage of avoiding lengthly Monte Carlo simulations. For brevity, the explicit expression of the first derivative of the MGF of the aggregate other-cell interference is not reported. It can be computed and formulated in closed-form from (13) and (14). The numerical complexity associated with the computation of Proposition 1 and Proposition 2 instead of using Monte Carlo simulations in discussed in Section VI 


\section{NumericAl AND Simulation Results}

In this section, we capitalize on Monte Carlo simulations in order to validate our findings and mathematical frameworks, as well as to assess the potential of the proposed A-SWIPT scheme for application to cellular networks. Monte Carlo simulations are obtained by using the approach discussed in [19]. Unless otherwise stated, the following setup is considered: $\nu=c_{0} / f_{c}$, where $c_{0}$ is the speed of light in $\mathrm{m} / \mathrm{s}$ and $f_{c}=2.1 \mathrm{GHz}$ is the carrier frequency; $\sigma_{\mathrm{ID}}^{2}=-70$ $\mathrm{dBm} ; \sigma_{N}^{2}=-174+10 \log _{10}\left(B_{w}\right)+\mathcal{F}_{N} \mathrm{dBm}$, where $B_{w}=200 \mathrm{kHz}$ and $\mathcal{F}_{N}=10 \mathrm{~dB}$ is the noise figure; $P=30 \mathrm{dBm} ; \zeta=0.8 ; \omega_{\mathrm{M}}=25.6$ degrees; $G_{\mathrm{M}}=7.47 ;$ and $G_{\mathrm{S}}=0.5$. The channel model and the density of BSs, $\lambda$, are chosen in agreement with [19]: $D=109.8517 \mathrm{~m}$,

$q_{\mathrm{LOS}}^{[0, D]}=0.7195, q_{\mathrm{LOS}}^{[D, \infty]}=0.0002, \beta_{\mathrm{LOS}}=2.5, \beta_{\mathrm{NLOS}}=3.5, \lambda=1 /\left(\pi R_{\text {cell }}^{2}\right)$ where $R_{\text {cell }}=83.4122$ $\mathrm{m}$ denotes the average cell radius. In Figs. 1-3, without loss of generality, we analyze the case study $\bar{F}\left(\mathcal{R}_{*}, \mathcal{Q}_{*}\right)=0.75$. Usually, imposing higher values of the J-CCDF results in lower values of $\mathcal{R}_{*}$ and $\mathcal{Q}_{*}$ that satisfy them. The setup $\bar{F}\left(\mathcal{R}_{*}, \mathcal{Q}_{*}\right)=0.75$ is considered only as an illustrative example. Our frameworks can be applied for arbitrary parameters and setups. In Fig. 4, in particular, we analyze a case study where $\bar{F}\left(\mathcal{R}_{*}, \mathcal{Q}_{*}\right)=0.9$, which corresponds to an application scenario where the imposed requirements of information rate and harvested power need to be achieved with high reliability.

In Figs. 1 and 2, we validate the correctness of the mathematical frameworks in Proposition 1 and Proposition 2, respectively, against Monte Carlo simulations. In particular, Fig. 1 confirms that the J-CCDF in Proposition 1 is exact and Fig. 2 highlights that the Frechet inequality in Proposition 2 provides a upper-bound of the J-CCDF, which is asymptotically tight as the system operates either in the ID-limited or in the EH-limited regimes. From the engineering standpoint, Figs. 1 and 2 show that information rate and harvested power highly depend on the choice of $\rho$ for PS and PS-MRC schemes, as well as that there is no scheme among SAR, ID-SC and EH-SC that outperform all the others for every pair $\left(\mathcal{R}_{*}, \mathcal{Q}_{*}\right)$. All lemmas and remarks in Section IV are, in particular, confirmed. This motivates the need of the proposed adaptive schemes for system-level optimization.

In Fig. 3, we investigate the performance of the proposed adaptive schemes and compare them against the ideal setups where ID and EH can be performed without any practical implementation 


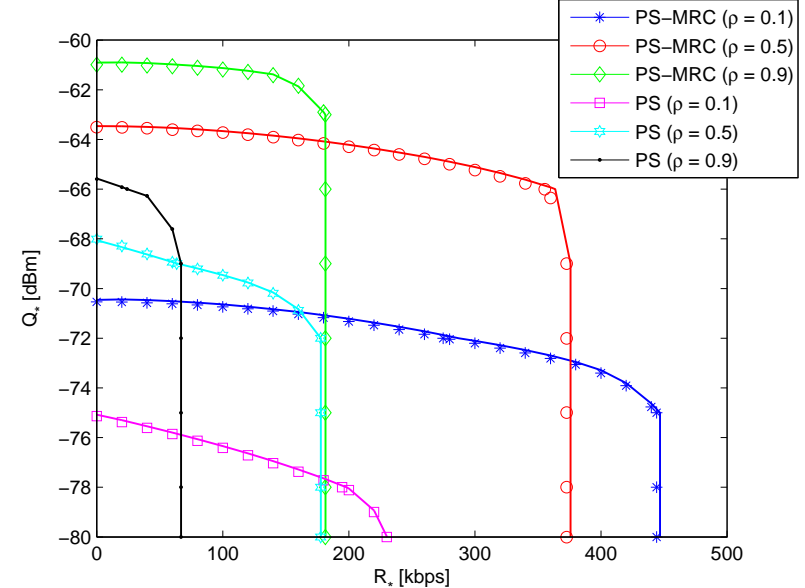

Fig. 1: Contour lines of the J-CCDF of PS and PSMRC schemes as a function of $\rho$. The curves show the pairs $\left(\mathcal{R}_{*}, \mathcal{Q}_{*}\right)$ so that $\bar{F}\left(\mathcal{R}_{*}, \mathcal{Q}_{*}\right)=0.75$. Markers: Monte Carlo simulations. Solid lines: Proposition 1 .

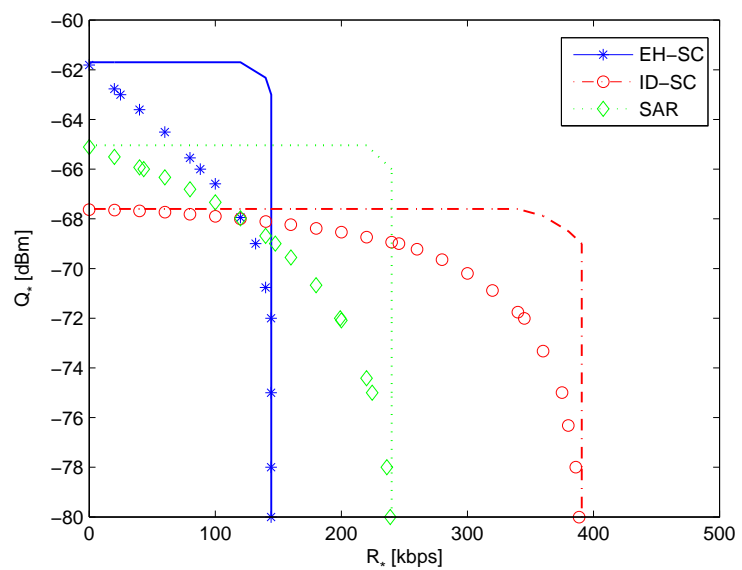

Fig. 2: Contour lines of the J-CCDF of SAR, ID$\mathrm{SC}$ and EH-SC schemes. The curves show the pairs $\left(\mathcal{R}_{*}, \mathcal{Q}_{*}\right)$ so that $\bar{F}\left(\mathcal{R}_{*}, \mathcal{Q}_{*}\right)=0.75$. Markers: Monte Carlo simulations. Solid, dotted and dashed lines: Proposition 2.

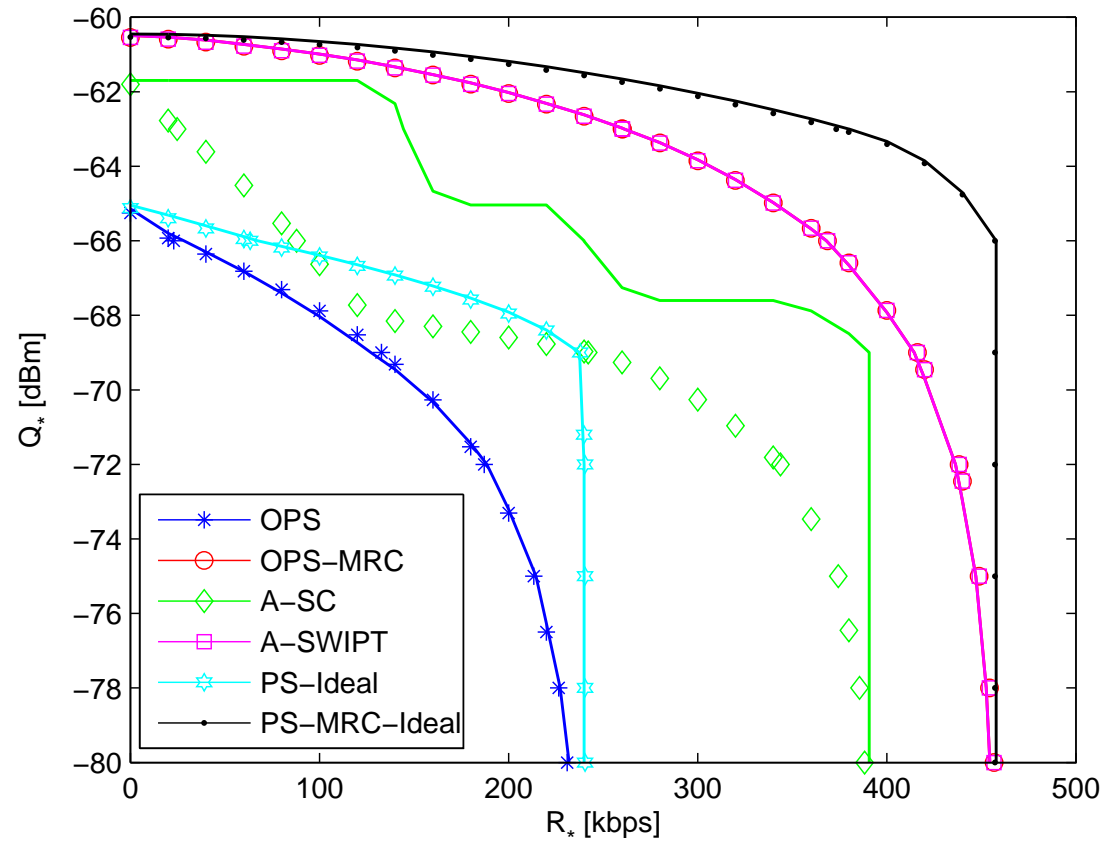

Fig. 3: Contour lines of the J-CCDF of OPS, OPS-MRC, A-SC and A-SWIPT schemes. The curves show the pairs $\left(\mathcal{R}_{*}, \mathcal{Q}_{*}\right)$ so that $\bar{F}\left(\mathcal{R}_{*}, \mathcal{Q}_{*}\right)=0.75$. The setups PS-Ideal and PS-MRC-Ideal refer to the PS and PS-MRC schemes where $\rho_{\mathrm{ID}}=0$ and $\rho_{\mathrm{EH}}=1$ simultaneously, e.g., ID and EH are assumed to be performed on the same signal without the need of PS. $\rho_{\text {opt }}$ is computed by using Proposition 1. Markers: Monte Carlo simulations. Solid lines: Proposition 1 for OPS, OPS-MRC and A-SWIPT schemes and in Proposition 2 for the A-SC scheme. In the considered setup, the OPS-MRC scheme coincides with the A-SWIPT scheme. 


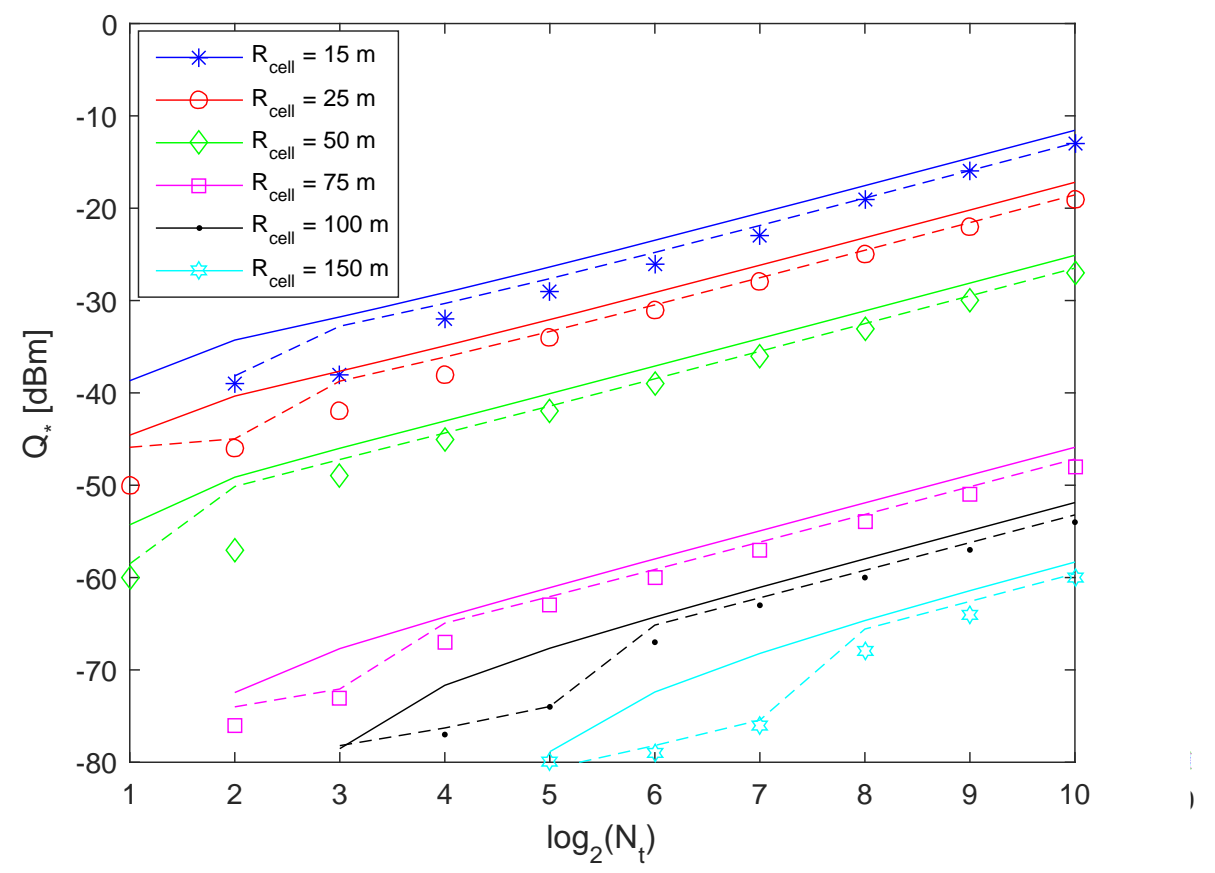

Fig. 4: Highest harvested power $\mathcal{Q}_{*}$ of OPS-MRC and A-SC so that $\bar{F}\left(\mathcal{R}_{*}, \mathcal{Q}_{*}\right)=0.90$ and $\mathcal{R}_{*}=100 \mathrm{kbps}$, as a function of $R_{\text {cell }} . \rho_{\text {opt }}$ is computed by using Proposition 1. Markers: Monte Carlo simulations for the A-SC scheme. Solid lines: Proposition 1 for the OPS-MRC scheme. Dashed lines: Proposition 2 for the A-SC scheme.

constraints (denoted by "Ideal" in the figure). We observe that the proposed A-SWIPT scheme outperforms all the other schemes, as well as that its J-CCDF is not far from the corresponding ideal benchmark. In the considered setup, in particular, we note that the J-CCDF of the ASWIPT scheme coincides with the J-CCDF of the OPS-MRC scheme. This implies that, in the considered setup, the OPS-MRC scheme outperforms the A-SC scheme for every pair $\left(\mathcal{R}_{*}, \mathcal{Q}_{*}\right)$.

In Fig. 4, we leverage the proposed mathematical frameworks for computing the highest power that can be harvested, $\mathcal{Q}_{*}$, for some given requirements of achievable rate, $\mathcal{R}_{*}$, and reliability formulated in terms of J-CCDF. The figure, in particular, highlights the impact of the density of BSs via $R_{\text {cell }}$ and of directional beamforming. More precisely, $N_{t}$ is the number of antennas of the directional beamformer in [18]. The values of $\omega_{M}, G_{M}$ and $G_{S}$ used in Figs. 1-3 can be obtained by setting $N_{t}=4$. The figure proves that the densification of BSs and antenna elements increases the amount of harvested power remarkably. We note, in particular, that $\mathcal{Q}_{*}$ increases almost linearly with the logarithm of $N_{t}$. In addition, two important performance trends can be identified: i) OPS-MRC and A-SC provide similar performance as $N_{t}$ increases, which highlights 
TABLE IV: Optimum power splitting ratio for different values of $\mathcal{Q}_{*}$ and $\mathcal{R}_{*}$ expressed in $\mathrm{dBm}$ and kbps, respectively. The "Exact" values are obtained by using Proposition 1. The "Upper-bound" values are obtained by using Proposition 2. The "Mixed" values are obtained by using Proposition 2 to compute $\rho_{\text {opt }}$ and by obtaining the corresponding J-CCDF by using Proposition 1.

\begin{tabular}{|c||c|c|c|c|c|}
\hline$\left(\mathcal{Q}_{*}, \mathcal{R}_{*}\right) \rightarrow$ & $(-60,100)$ & $(-60,200)$ & $(-60,300)$ & $(-60,400)$ & $(-60,500)$ \\
\hline \multirow{2}{*}{ Exact } & $\rho_{\text {opt }}=0.9568$ & $\rho_{\text {opt }}=0.9108$ & $\rho_{\text {opt }}=0.8526$ & $\rho_{\text {opt }}=0.7825$ & $\rho_{\text {opt }}=0.7004$ \\
& $\bar{F}_{\text {opt }}=0.7447$ & $\bar{F}_{\text {opt }}=0.7401$ & $\bar{F}_{\text {opt }}=0.7334$ & $\bar{F}_{\text {opt }}=0.7243$ & $\bar{F}_{\text {opt }}=0.7121$ \\
\hline \multirow{2}{*}{ Upper-bound } & $\rho_{\text {opt }}=0.9640$ & $\rho_{\text {opt }}=0.9036$ & $\rho_{\text {opt }}=0.8006$ & $\rho_{\text {opt }}=0.6352$ & $\rho_{\text {opt }}=0.4208$ \\
& $\bar{F}_{\text {opt }}=0.7465$ & $\bar{F}_{\text {opt }}=0.7452$ & $\bar{F}_{\text {opt }}=0.7425$ & $\bar{F}_{\text {opt }}=0.7375$ & $\bar{F}_{\text {opt }}=0.7280$ \\
\hline \multirow{2}{*}{ Mixed } & $\rho_{\text {opt }}=0.9640$ & $\rho_{\text {opt }}=0.9036$ & $\rho_{\text {opt }}=0.8006$ & $\rho_{\text {opt }}=0.6352$ & $\rho_{\text {opt }}=0.4209$ \\
& $\bar{F}_{\text {opt }}=0.7446$ & $\bar{F}_{\text {opt }}=0.7401$ & $\bar{F}_{\text {opt }}=0.7327$ & $\bar{F}_{\text {opt }}=0.7213$ & $\bar{F}_{\text {opt }}=0.7032$ \\
\hline \hline$\left(\mathcal{Q}_{*}, \mathcal{R}_{*}\right) \rightarrow$ & $(-80,100)$ & $(-70,100)$ & $(-60,100)$ & $(-50,100)$ & $(-40,100)$ \\
\hline \multirow{2}{*}{ Exact } & $\rho_{\text {opt }}=0.1983$ & $\rho_{\text {opt }}=0.7166$ & $\rho_{\text {opt }}=0.9568$ & $\rho_{\text {opt }}=0.99$ & $\rho_{\text {opt }}=0.99$ \\
\hline \multirow{2}{*}{ Upper-bound } & $\bar{F}_{\text {opt }}=0.9051$ & $\bar{F}_{\text {opt }}=0.8213$ & $\bar{F}_{\text {opt }}=0.7447$ & $\bar{F}_{\text {opt }}=0.6333$ & $\bar{F}_{\text {opt }}=0.2028$ \\
\hline \multirow{2}{*}{ Mixed } & $\bar{F}_{\text {opt }}=0.9084$ & $\rho_{\text {opt }}=0.7103$ & $\rho_{\text {opt }}=0.9640$ & $\rho_{\text {opt }}=0.99$ & $\rho_{\text {opt }}=0.99$ \\
& $\rho_{\text {opt }}=0.1669$ & $\bar{F}_{\text {opt }}=0.7263$ & $\bar{F}_{\text {opt }}=0.7465$ & $\bar{F}_{\text {opt }}=0.6348$ & $\bar{F}_{\text {opt }}=0.2049$ \\
\hline
\end{tabular}

that SC may be a low-complexity option with minimal performance degradation with respect to MRC and ii) the mathematical framework in Proposition 2 is in good agreement with Monte Carlo simulations. It is worth mentioning that the values of $N_{t}$ for which the J-CCDF does not reach 0.9 are not shown in the figure.

In Table IV, we analyze the accuracy of computing the optimum power splitting ratio, $\rho$, by using the upper-bound in Proposition 2. This study is motivated by the comment in Remark 3. The numerical results confirm that, even though the upper-bound may provide slightly different values of $\rho_{\text {opt }}$, its accuracy is usually acceptable and the corresponding values of the J-CCDF are sufficiently close to those obtained with using the exact optimum power splitting ratio.

In Table $\mathrm{V}$, we provide numerical examples for substantiating the comment made right after Lemma 6, i.e., PS may outperform PS-MRC for values of $\rho$ that do not satisfy the conditions stated in the lemma. The reason of this performance trend, which may be considered to be unexpected at the first sight, is related to the fact that PS and PS-MRC are configured to operate in different regimes. Due to the small and large values of the power splitting ratios, PS and PS-MRC operate close to the ID-limited and EH-limited regimes, respectively. This implies that their J-CCDFs have different mathematical expressions and, thus, the impact of MRC cannot be predicted based on conventional arguments. This motivates the need and relevance of the comparison and findings summarized in Lemma 6. The values reported in the third column of 
TABLE V: J-CCDF of PS and PS-MRC schemes as a function of $\mathcal{Q}_{*}$ and $\mathcal{R}_{*}$ expressed in $\mathrm{dBm}$ and kbps, respectively. Setup: $\rho=0.001$ for PS and $\rho=0.999$ for PS-MRC.

\begin{tabular}{|c||c|c|c|c|c|}
\hline$\left(\mathcal{Q}_{*}, \mathcal{R}_{*}\right) \rightarrow$ & $(-60,800)$ & $(-60,900)$ & $(-60,944.144)$ & $(-60,950)$ & $(-60,1050)$ \\
\hline PS & 0.020811 & 0.0207128 & 0.0206575 & 0.0206493 & 0.0204861 \\
\hline PS-MRC & 0.0309 & 0.0232655 & 0.0205 & 0.0202259 & 0.0151995 \\
\hline \hline$\left(\mathcal{Q}_{*}, \mathcal{R}_{*}\right) \rightarrow$ & $(-65,500)$ & $(-65,600)$ & $(-65,630)$ & $(-65,700)$ & $(-65,800)$ \\
\hline PS & 0.0505034 & 0.0502939 & 0.0502159 & 0.0499982 & 0.0495784 \\
\hline PS-MRC & 0.0758926 & 0.0556419 & 0.0508347 & 0.0413108 & 0.0309119 \\
\hline \hline$\left(\mathcal{Q}_{*}, \mathcal{R}_{*}\right) \rightarrow$ & $(-70,300)$ & $(-70,350)$ & $(-70,366.15)$ & $(-70,400)$ & $(-70,450)$ \\
\hline PS & 0.119039 & 0.118727 & 0.118614 & 0.118369 & 0.117965 \\
\hline PS-MRC & 0.151332 & 0.125689 & 0.118636 & 0.105478 & 0.0891926 \\
\hline \hline$\left(\mathcal{Q}_{*}, \mathcal{R}_{*}\right) \rightarrow$ & $(-75,100)$ & $(-75,150)$ & $(-75,174.76)$ & $(-75,200)$ & $(-75,250)$ \\
\hline PS & 0.261551 & 0.260555 & 0.260041 & 0.259499 & 0.258374 \\
\hline PS-MRC & 0.397323 & 0.295535 & 0.259963 & 0.230231 & 0.184795 \\
\hline \hline$\left(\mathcal{Q}_{*}, \mathcal{R}_{*}\right) \rightarrow$ & $(-80,20)$ & $(-80,70)$ & $(-80,75.674)$ & $(-80,80)$ & $(-80,130)$ \\
\hline PS & 0.470646 & 0.468389 & 0.468131 & 0.467937 & 0.465607 \\
\hline PS-MRC & 0.685975 & 0.487832 & 0.468606 & 0.454633 & 0.330482 \\
\hline
\end{tabular}

TABLE VI: Comparison of the time (in seconds) for computing the J-CCDF via Monte Carlo simulations and Proposition 1, for different pairs $\left(\mathcal{Q}_{*}, \mathcal{R}_{*}\right)$ in $(\mathrm{dBm}, \mathrm{kpbs})$. As for Monte Carlo simulations, the number of spatial and channel realizations is set equal to 1000 in all setups, while the simulation area, Area, (in square meters) is chosen so that the relative error, Error, between the J-CCDF functions computed with Proposition 1 and estimated via simulation have a relative error equal to around $0.1 \%$. For completeness, the relative error (in \%) is reported as well. The time for computing $\rho_{\text {opt }}$ is not taken into account.

\begin{tabular}{|c||l|l|l|l|l|}
\hline$\left(\mathcal{Q}_{*}, \mathcal{R}_{*}\right) \rightarrow$ & $(-63,200)$ & $(-66,300)$ & $(-69,400)$ & $(-75,400)$ & $(-78,400)$ \\
\hline \hline \multirow{3}{*}{ Monte Carlo } & Time $=1761$ & Time $=294$ & Time $=1782$ & Time $=304$ & Time $=1547$ \\
& $\bar{F}(\cdot)=0.7540$ & $\bar{F}(\cdot)=0.7599$ & $\bar{F}(\cdot)=0.7534$ & $\bar{F}(\cdot)=0.7617$ & $\bar{F}(\cdot)=0.7630$ \\
& Area $=2.1858 e 9$ & Area $=2.1858 e 8$ & Area $=2.1858 e 9$ & Area $=2.1858 e 8$ & Area $=2.1858 e 9$ \\
& Error $=0.1049$ & Error $=0.0996$ & Error $=0.0904$ & Error $=0.1156$ & Error $=0.0906$ \\
\hline \multirow{2}{*}{ Framework } & Time $=83$ & Time $=63$ & Time $=71$ & Time $=65$ & Time $=63$ \\
& $\bar{F}(\cdot)=0.754792$ & $\bar{F}(\cdot)=0.759144$ & $\bar{F}(\cdot)=0.7527194$ & $\bar{F}(\cdot)=0.7606211$ & $\bar{F}(\cdot)=0.76230968$ \\
& $\rho_{\text {opt }}=0.843616$ & $\rho_{\text {opt }}=0.60452875$ & $\rho_{\text {opt }}=0.3198705$ & $\rho_{\text {opt }}=0.106576097$ & $\rho_{\text {opt }}=0.05698357663$ \\
\hline
\end{tabular}

Table $\mathrm{V}$, in particular, correspond to the pair $\left(\mathcal{Q}_{*}, \mathcal{R}_{*}\right)$ for which PS and PS-MRC provide almost the same J-CCDF.

In Table VI, we compare the proposed mathematical frameworks and Monte Carlo simulations in terms of the computation time that is necessary for estimating the J-CCDF for a single pair $\left(\mathcal{Q}_{*}, \mathcal{R}_{*}\right)$. Since the computation time of Monte Carlo simulations highly depends on the simulation area, which determines the accuracy of the result, the study is conducted by setting the simulation area so that the relative error between the J-CCDF computed with the mathematical framework and that estimated via Monte Carlo simulations is around $0.1 \%$. It is worth mentioning that Table VI does not account for the time needed to compute the optimal power splitting ratio, $\rho_{\text {opt }}$. This computation is, in fact, not affordable by using Monte Carlo simulations due to the 
many possible values that need to be analyzed. Even neglecting this latter significant computation time, we note that the proposed mathematical frameworks are faster than Monte Carlo simulations for values of the simulation area that provide sufficiently accurate estimates of the J-CCDF. This justifies even further the usefulness of the proposed mathematical frameworks: they are not only insightful and make our numerical illustrations easier to be reproduced (reproducible research), but are more numerically tractable as well.

\section{CONCLUSION}

In this paper, we have analyzed SWIPT-enabled cellular networks that employ several receiver diversity schemes. We have shown that receiver diversity has the potential of enhancing the information rate and of increasing the harvested power simultaneously. We have proved, in addition, that the system-level performance can be improved by adaptively choosing the receiver diversity scheme as a function of the information rate and of the harvested power requirements that need to be fulfilled. With the aid of stochastic geometry, we have introduced mathematical frameworks that enable one to perform this system-level and adaptive optimization. All findings and performance trends have been validated with the aid of Monte Carlo simulations.

As far as the SWIPT schemes that use selection combining are concerned, the analysis has been based on a upper-bound that exploits the Frechet inequality. This proposed bound provides several important design guidelines for system-level optimization and it is asymptotically tight in the ID-limited regime and in the EH-limited regime. In general, however, a gap between the exact J-CCDF and its estimate based on the upper-bound exists (see Figs. 2 and 3). The authors are currently working on the development of more accurate, but still tractable, bounds and approximations for arbitrary information rate and harvested power requirements.

\section{APPENDIX A}

\section{ON THE EXACT J-CCDF OF SAR, ID-SC AND EH-SC SWIPT SCHEMES}

In Section V, it is argued that an exact expression for the J-CCDF of SAR, ID-SC and EH-SC schemes may be obtained with the aid of the multi-dimensional inversion theorem [21], which, however, would result in a multi-fold integral expression that is much less tractable than the 
mathematical framework reported in Proposition 2. In this appendix, we provide further details on how this framework can be developed. Due to space limitations, no formulas are reported but only the approach is briefly summarized.

The approach is along the same lines as the proof of Proposition 1. More precisely, the JCCDF can be formulated as shown in the first line of (18). In this case, however, the other-cell interferences of ID and EH receiver are different as discussed in Remark 1. As a result, the equality in the second line of (18) does not hold anymore, since it assumes that the other-cell inferences are the same. Nevertheless, the joint CF of the other-cell interferences of ID and EH receivers can be computed by using the same steps as in [18, Lemma 2] and the associated joint CDF can be formulated in terms of the joint CF by using [21, Eq. (11)]. The resulting expression, however, necessitates the computation of a two-fold integral. Since the computation of the expectation with respect to the smallest path-loss (see (18)) requires another integral, the final expression of the J-CCDF may require the computation of at least a three-fold integral. This makes the resulting mathematical framework less tractable and less numerically stable than the upper-bound in Proposition 2. This motivates the use of the Frechet bound in (7).

\section{REFERENCES}

[1] A. Al-Fuqaha, M. Guizani, M. Mohammadi, M. Aledhari, and M. Ayyash, "Internet of things: A survey on enabling technologies, protocols, and applications”, IEEE Commun. Surveys \& Tuts., vol. 17, no. 4, pp. 2347-2376, 2015.

[2] I. Krikidis, S. Timotheou, S. Nikolaou, G. Zheng, D. W. K. Ng, and R. Schober, "Simultaneous wireless information and power transfer in modern communication systems", IEEE Commun. Mag., vol. 52, no. 11, pp. 104-110, Nov. 2014.

[3] http://vandrico.com/wearables/device/intel-mica.

[4] http://www.cnet.com/news/ring-ring-its-your-watch-calling-att-bets.

[5] http://www.3gpp.org/news-events/3gpp-news/1733-niot.

[6] M. Di Renzo, A. Guidotti, and G. E. Corazza, "Average rate of downlink heterogeneous cellular networks over generalized fading channels - A stochastic geometry approach", IEEE Trans. Commun., vol. 61, no. 7, pp. 3050-3071, July 2013.

[7] G. Zheng, I. Krikidis, C. Masouros, S. Timotheou, D. Toumpakaris, and Z. Ding, "Rethinking the role of interference in wireless networks", IEEE Commun. Mag., vol. 52, no. 11, pp. 152-158, Nov. 2014.

[8] Z. Ding, C. Zhong, D. W. K. Ng, M. Peng, H. A. Suraweera, R. Schober, and H. V. Poor, "Application of smart antenna technologies in simultaneous wireless information and power transfer”, IEEE Commun. Mag., vol. 53, no. 4, pp. 86-93, Apr. 2015.

[9] M. Di Renzo, H. Haas, A. Ghrayeb, S. Sugiura, and L. Hanzo, "Spatial modulation for generalized MIMO: Challenges, opportunities and implementation," Proc. of the IEEE, vol. 102, no. 1, pp. 56-103, Jan. 2014. 
[10] L. Liu, R. Zhang, and K. C. Chua, "Wireless information and power transfer: A dynamic power splitting approach", IEEE Trans. Commun., vol. 61, vol. 9, pp. 3990-4001, Sep. 2013.

[11] I. Krikidis, S. Sasaki, S. Timotheou, and Z. Ding, "A low complexity antenna switching for joint wireless information and energy transfer in MIMO relay channels", IEEE Trans. Commun., vol. 62, vol. 5, pp. 1577-1587, May 2014.

[12] X. Zhou, R. Zhang, and C. Ho, "Wireless information and power transfer: Architecture design and rate-energy tradeoff", IEEE Trans. Commun., vol. 61, vol. 11, pp. 4754-4767, Nov. 2013.

[13] I. Krikidis, "Simultaneous information and energy transfer in large-scale networks with/without relaying", IEEE Trans. Commun., vol. 62, vol. 3, pp. 900-912, Mar. 2014.

[14] Z. Ding, I. Krikidis, B. Sharif, and H. V. Poor, "Wireless information and power transfer in cooperative networks with spatially random relays", IEEE Trans. Wireless Commun., vol. 13, no. 8, pp. 4440-4453, Aug. 2014.

[15] J. Guo, S. Durrani, X. Zhou, and H. Yanikomeroglu, "Outage probability of ad hoc networks with wireless information and power transfer", IEEE Wireless Commun. Lett., vol. 4, no. 4, pp. 409-412, Aug. 2015.

[16] I. Flint, L. Xiao Lu, N. Privault, D. Niyato, and P. Wang, "Performance analysis of ambient RF energy harvesting with repulsive point process modeling”, IEEE Trans. Wireless Commun., vol. 14, no. 10, pp. 5402-5416, Oct. 2015.

[17] X. Zhou, J. Guo, S. Durrani, and I. Krikidis, "Performance of maximum ratio transmission in ad hoc networks with SWIPT”, IEEE Wireless Commun. Lett., vol. 4, no. 5, pp. 529-532, Oct. 2015.

[18] W. Lu, M. Di Renzo, and T. Q. Duong, “On stochastic geometry analysis and optimization of wireless-powered cellular networks", in Proc. IEEE GLOBECOM, Dec. 2015, pp. 1-6.

[19] W. Lu and M. Di Renzo, "Stochastic geometry modeling of cellular networks: Analysis, simulation and experimental validation", in Proc. ACM MSWiM, Nov. 2015, pp. 179-188.

[20] M. Fréchet, "Sur les tableaux de corrélation dont les marges son donnés", Annales de l'Université de Lyon, Série 3, vol. 4, pp. 53-57, 1951.

[21] N. G. Shephard, "From characteristic function to distribution function: A simple framework for the theory", Econometric theory, vol. 7, no. 4, pp. 519-529, 1991.

[22] M. Di Renzo and P. Guan, "Stochastic geometry modeling of coverage and rate of cellular networks using the Gil-Pelaez inversion theorem”, IEEE Commun. Lett., vol. 18, no. 9, pp. 1575-1578, Sep. 2014. 